\title{
Cascading Failures in Weighted Complex Networks of Transit Systems Based on Coupled Map Lattices
}

\author{
Ailing Huang, ${ }^{1,2}$ H. Michael Zhang, ${ }^{2}$ Wei Guan, ${ }^{1}$ Yang Yang, ${ }^{1}$ and Gaoqin Zong ${ }^{1}$ \\ ${ }^{1}$ MOE Key Laboratory for Urban Transportation Complex Systems Theory and Technology, Beijing Jiaotong University, \\ Beijing 100044, China \\ ${ }^{2}$ Department of Civil and Environment Engineering, University of California, Davis, CA 95616, USA
}

Correspondence should be addressed to H. Michael Zhang; hmzhang@ucdavis.edu

Received 24 April 2014; Revised 4 July 2014; Accepted 4 July 2014

Academic Editor: José R. C. Piqueira

Copyright (C) 2015 Ailing Huang et al. This is an open access article distributed under the Creative Commons Attribution License, which permits unrestricted use, distribution, and reproduction in any medium, provided the original work is properly cited.

\begin{abstract}
Study on the vulnerability and robustness of urban public transit networks (PTNs) has great implications for PTNs planning and emergency management, particularly considering passengers' dynamic behaviors. We made a complex weighted network analysis based on passenger flow for Beijing's bus stop network and multimodal transit network coupled with bus and urban rail systems. The analysis shows that there are small-world or scale-free properties in these two networks, which make them display different robustness under link or node failures. With consideration of the dynamic flow redistribution, we propose a model based on coupled map lattices to analyze the cascading failures of these two weighted networks. We find that the dynamic flow redistribution can significantly improve the tolerance of small-world or scale-free PTN against random faults. Because of the coupling of bus and rail systems, the multimodal network with scale-free topology and flow distribution structures displays an increasing tolerance even against intentional attack; however, its cascade is also much more intense once the failure is triggered. We find some thresholds of topological and flow coupling strength in the spreading process, which can be exploited to develop strategies to control cascade failures.
\end{abstract}

\section{Introduction}

In the last few years, the error tolerance and attack vulnerability of real networks have attracted the attention of many researchers, since the discovery of the ubiquitous small-world [1] and scale-free properties [2]. A scale-free network displays a property of heterogeneity with a powerlaw degree distribution $p(k) \sim k^{-\gamma}$ [2], where $k$ is the degree of a randomly chosen node and $\gamma$ is the scale-free exponent, while a small-world network, compared to the former, is more homogeneous with an exponential degree distribution, a greater clustering coefficient, and a shorter average distance [1]. Some empirical studies report that many real networks' topological structures, including public transit networks (PTNs), are found to be scale-free or small-world, for example, the Boston subway network $[3,4]$, the Indian railway network [5], the Chinese railway network [6], and the public transport systems in Poland [7], in Germany, and in France [8]. The scale-free networks become "robust yet fragile" $[9,10]$; that is, these networks are very sensitive to the intentional removal of central nodes but become robust to the random removal of some other nodes. There have been a few recent empirical studies on the structural robustness of many real complex networks, for example, the protein network [11], the food webs [12], the email network [13], the internet network $[14,15]$, the computer network [16], the cyber-physical network [17], and the uncertain system $[18,19]$.

In addition, there exists the physical flow (also defined as load) in real-world networks, such as the data transmitted in communication networks, the electrical currents in power grids, the vehicles in transportation networks, and the passenger flow in PTNs. As the physical flow is dynamic, the failure of one or a few nodes/edges could lead to the failures of other nodes/edges by the coupling relationship between nodes/edges and the redistribution of flow over the whole networks, triggering chain reactions that lead to the collapse of a few or all nodes/edges in the network. This 
phenomenon is called cascading failure [20,21]. The largest blackout event in the northeastern US power grid in August 2003, for example, is due to cascading failure.

Urban PTN, composed of routes, stops, and the passenger flow traveling in network, is a complicated giant system. In a PTN, if one or some key stations or routes are destroyed by natural disasters or their carrying capacity is in short supply due to sudden increases of passenger flow (such as a large number of people coming from stadiums or theaters after matches or concerts), one part of, or even the whole, network may be overloaded and out of operation. A case like this occurred on July 21, 2012, when the heaviest rain in the last 60 years in Beijing shut down a metro line and caused 100 bus routes to detour, dump stop, or stop operation completely. This resulted in more than 200 bus routes extending their service hours to transport passengers. Another example occurred on January 3, 2010, when heavy snow caused more than 60 bus routes to detour or be taken out of operation. These failures greatly impacted passenger travel and severely disrupted the social/economic activities of the city. These events motivated us to study the dependence of avalanches and dynamic robustness in PTNs on the initial perturbation, network structure, and flow behavior.

Some models and empirical research on cascading failures in different networks have been reported in the recent literature, and many of them showed that a network's structure has great impact on its function. They include the empirical studies on the US power grid [22] and ecoindustrial symbiosis network considering the load redistribution, the cascading dynamics in small-world network [23, 24], scalefree network [25], weighted complex network [26], and independent network [27]. For PTNs, however, the majority of the research focuses on the (static) robustness of network topology based on the idea of Albert et al. [9], by removing one or some nodes or edges in the network. A typical example is the analysis of attack vulnerability of 14 PTNs in the Land P-spaces (explained in Figure 1) [28, 29], respectively. Topological vulnerability of other PTN networks, such as the railway network of Switzerland [30], the subway network of Shanghai [31], the air flight network of China [32], is also studied.

The research on the dynamic robustness of PTNs, however, is still limited. Although Wu et al. [33,34] and Zheng et al. [35] have studied the cascading behavior in scale-free networks based on the traffic dynamics theory and Yang et al. [36] have simulated the epidemic dynamic behaviors in PTNs based on the SIS spread model, the cascading dynamics in weighted PTNs considering the dynamical passenger flow has not yet been reported. Also, the empirical studies on the dynamical characteristics of weighted PTNs based on passenger's travel behavior are not given adequate consideration. As far as we know, only the studies of the Seoul subway system [37], Singapore public transportation system [38], and Beijing bus routes system [39] considered passenger flow in their analysis. The former two studies constructed the complex network according to passengers' travel routes, but the underlying physical structure is not considered.

In light of the review, several questions remain open in the study of PTNs considering flow dynamics. For example, what is the structure of a real PTN measured in the P-space [4] or L-space [5]? How robust are these PTNs against random fault or intentional attack when considering passenger flow redistribution in the network? What are the thresholds for cascading failures and spreading dynamics in different realworld PTN structures? In seeking answers to these questions, we were able to identify the threshold to avoid cascading failures in PTNs and find the strategies to avoid such failures, which provides an important theoretical basis for protecting real PTNs and improving their robustness.

In this paper, we propose a model to explore the dynamic robustness in actual PTNs of Beijing considering passenger flow behavior. Here, we study not only the bus stop network (BSN), but also the multimodal public transit network (MPTN) coupled with bus and urban rail transit systems, which will help us compare the PTNs' robustness with different weighted structures. The paper is organized as follows. In the next section, we will make an analysis of the statistical properties of the weighted complex network of Beijing's BSN and MPTN in L-space. In Section 3, a cascading failure analysis model for weighted network based on coupled map lattices is proposed, and, in Sections 4 and 5 , the dynamic robustness of BSN and MPTN in Beijing is studied, respectively. Conclusions and outlook are given in Section 6.

\section{Statistical Properties of Weighted Complex Network Derived from Beijing Public Transit System in L-Space}

2.1. Data Processing and Networks Generation. In this work, the weighted complex network for public transit stops in Beijing's bus and rail transit networks is built to explore the impact of connectivity between stops on passenger flow distribution. Here, we use the L-space method [5] to describe the network; that is, nodes represent bus, trolley bus, or rail stops, and a link between two nodes exists if they are consecutive stops on a route. The weight $w_{i j}$ between node $i$ and node $j$ is represented by the sectional passenger flow $P_{i j}$ that represents the number of passengers through the section between stop $i$ and adjacent stop $j$ in a weekday. Although the passenger flow is directed, in general, the daily flow is bidirectionally balanced for a whole day. For simplicity, we make the assumption that passenger flow is undirected $\left(w_{i j}=\right.$ $w_{j i}$ ) by averaging the in and out edge weights.

In Beijing's bus transit network, a flat fare system is applied in most of bus routes, which means commuters only swipe the smart card one time when they board the buses, so it is difficult to capture the exact data about sectional occupational passengers through the intelligent card (IC) system. A good way to acquire this information is by the ride check. We obtained the ride-check data of 100 routes in a weekday from Beijing Public Transportation Corporation. These routes are so typical that they were selected to investigate, and, on each route, the occupational passengers of bus in each section along the route for more than 200 bus trips per day were counted. According to data of 100 routes, stops, and sectional passengers in Beijing, we develop a weighted 


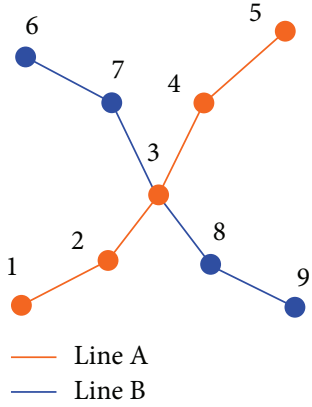

(a) L-space

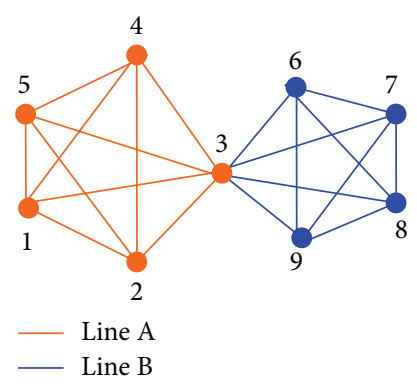

(b) P-space

Figure 1: Explanation of L-space (a) and P-space (b). Refer to [7].

bus stops network in the L-space with 1,388 nodes and 1,555 edges.

In the MPTN both for bus and rail stops, based on following methodology, the sectional passenger flow between two adjacent rail stops will be calculated in terms of passengers in and out of rail stops that are recorded by the IC system.

Firstly, we use the gravity model to estimate the origin and destination (OD) flow $q_{i j}$ from stop $i$ to $j$ :

$$
q_{i j}=a O_{i} D_{j} f\left(x_{i j}\right) \text {, }
$$

where $a$ is the trip generation coefficient; $O_{i}$ is the passengers going into stop $i ; D_{j}$ is the passengers out from stop $j ; f\left(x_{i j}\right)$ denotes an impedance function from stop $i$ to stop $j ; x_{i j}$ is an impedance parameter. As a flat fare is also applied in the whole urban rail network without any transfer fee, here, $x_{i j}$ is represented by the travel times between stops $i$ and $j$, composed of the train running time, dwell time in stops, and transfer time in transfer stations. The train running time is estimated based on the distance between stops and average train running velocity; the dwell time and transfer time are obtained by historical data and field investigations, respectively.

Secondly, considering the travel times to be the impedance, we assign the OD flow between stops into the rail network by all-or-nothing assignment through the TransCAD (TransCAD is a commercial software package that performs various types of traffic assignment) software, and then the sectional passenger flow between adjacent stops is calculated. Thus, based on the topology and flow data of 13 rail lines and 100 bus routes, the weighted complex MPTN in the L-space is established with 1,504 nodes and 1,728 edges.

2.2. Degree Distribution. In the L-space, the degree $k$ of a node represents the number of its consecutive neighbor stops it is linked to. It is calculated that the degree interval of MPTN in Beijing is $k_{B+R} \in[1,10]$, which is wider than that of BSN $k_{B} \in[1,8]$, although their average degrees are quite similar, $\left\langle k_{B}\right\rangle=2.24$ and $\left\langle k_{B+R}\right\rangle=2.29$, indicating that the connectivity of MPTN has been improved in part because of the addition of the rail system. As shown in Figure 2(a), the degree distribution of BSN appears exponentially distributed $p(k) \sim 0.4636 e^{-0.3286 k}$ and thus is small-world, which is consistent with Sui's PTN analysis [40] and similar to the degree distribution exponent of the North American power grid; that is, $\gamma \approx 0.5$ [4]. However, for the MPTN (shown in Figure 2(b)), its degree distribution plot is nearly a straight line on a log-log scale (red dots) and follows a power-law distribution $p(k) \sim 10.67 k^{-4.042}$. So, the MPTN is scalefree after the bus stops network is coupled with the rail network. It shows that the topology of Beijing's MPTN is much more heterogeneous than BSN, and some nodes are highly concentrated due to the limit of two-dimensional geographic space. This can be explained by the fact that the "rich get richer" phenomenon exists in PTNs just because bus routes tend to connect with rail stops.

2.3. Strength Distribution. The strength $s_{i}$ of node $i$ is defined as $s_{i}=\sum_{j} w_{i j}$ [41]; thus, a stop's strength in a PTN represents the average daily passenger flow passing the sections between this stop and its connected neighboring stops. Likewise, because of the rail system, the MPTN possesses wider node's strength interval $s_{B+R} \in[80,1.056343 \mathrm{e}+06]$ and higher average strength $\left\langle s_{B+R}\right\rangle=39500$ than that of BSN; $s_{B} \in$ $[64,60918]$ and $\left\langle s_{B}\right\rangle=9320$. As shown in Figure 3, there are long tails for strength distribution (blue dots) in both BSN and MPTN, especially in the latter, indicating that some stops transport quite large volume of passengers every day, which is much more than that of most stops. It is noted that the stop with large traffic may differ from the one with large degree. Not considering some stops with very small strengths in the BSN, both the strength distributions of BSN and MPTN on log-log scales are nearly linear and appear to follow powerlaw distributions $p(s) \sim c s^{-\gamma}$ with $\gamma=1.42$ and $\gamma=1.209$, respectively. The MPTN's power-law exponent is smaller than that of BSN, which reveals that the heterogeneity of MPTN has been enhanced when the bus network is coupled with the rail network. The strong heterogeneity of MPTN indicates that there are a few nodes called hubs in the network that have higher strength than other nodes and play a dominant role in preserving connections and serving traffic of the overall network. Such heterogeneity makes the scale-free network more robust to random failures but more vulnerable to deliberate attacks [9].

In summary, we find that both the topology and flow distribution structures of MPTN show the scale-free features 


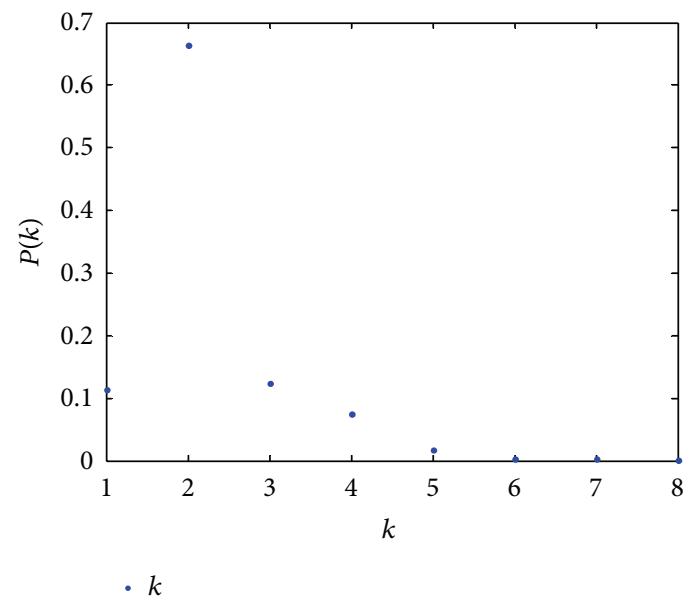

(a) BSN degree distribution

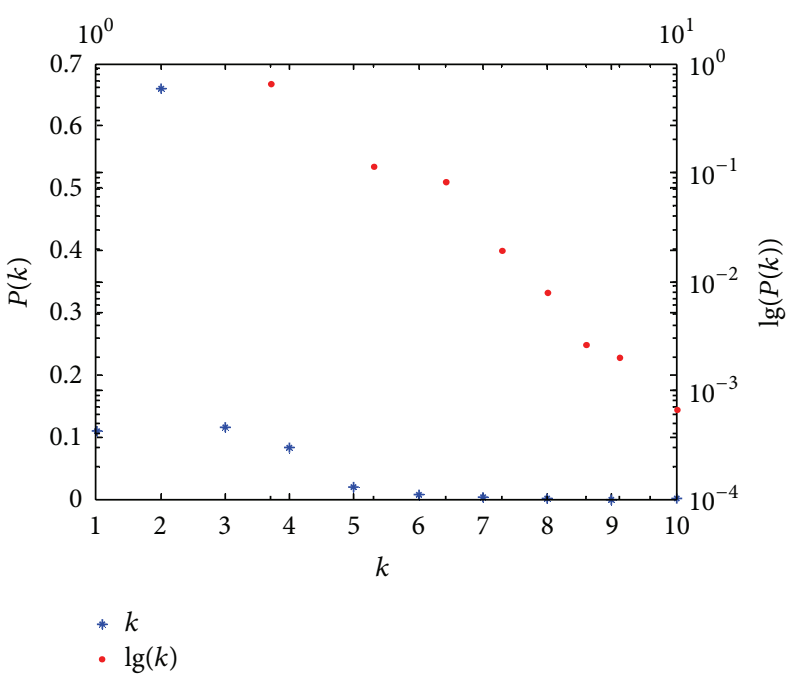

(b) MPTN degree distribution and its log-log scale plot

Figure 2: Node degree distribution for Beijing's BSN and MPTN.

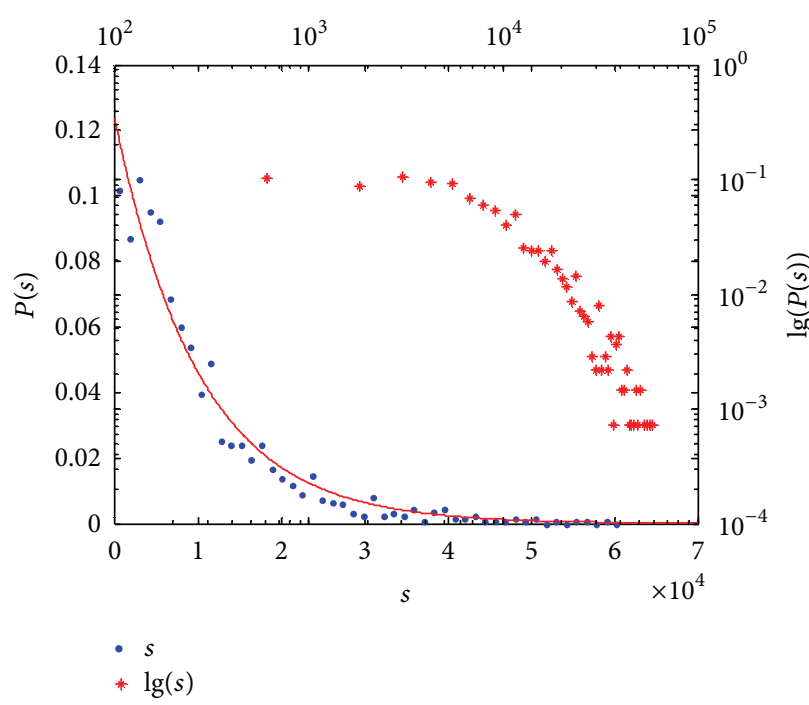

(a) BSN strength distribution

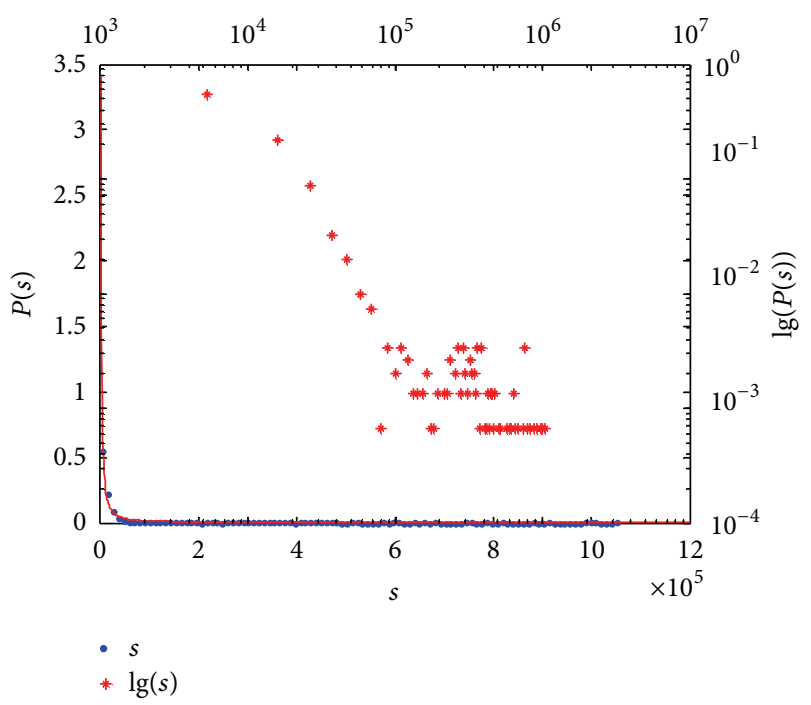

(b) MPTN strength distribution

FIgURE 3: Node's strength distribution of Beijing's weekday weighted BSN and MPTN and their log-log scale plot.

while the BSN is coupled with small-world topology and scale-free flow distribution, which could make them display different tolerance and robustness under random fault or intentional attack, so they will be further studied in the following sections.

\section{A Cascading Failure Analysis Based on Coupled Map Lattices}

3.1. A CMLs Model for Weighted Complex Network. A coupled map lattice (CML) is a dynamical system that models the behavior of nonlinear systems (especially partial differential equations) [42]. They are predominantly used to qualitatively study the chaotic dynamics of spatially extended systems.
Studied systems include populations, convection, fluid flow, chemical reactions, and biological networks. More recently, CMLs have been applied to computational networks identifying detrimental attack methods and cascading failures. However, the current literature of cascading failure in CMLs is almost based on topological network [43-48], and there is few research on static weighted network $[49,50]$, not to mention ones in view of dynamic weight. In fact, in the real world, not only topology but also the flow distribution influences the cascading failure of the network. It is often to see that when the flow is beyond the carrying capacity, cascading failures occur, for example, in the urban transportation network, the Internet, and the power grid network. There are also similar cascading failures in a PTN; namely, 
the redistribution of passenger flow caused by failures in one or a few stops or sections could lead to overload or outage of other nodes and then result in the whole network's failure. In a PTN, failures may be triggered by the change of network's topology or the redistribution of passenger flow, whereas, when failures occur, the topology and passenger flow are affected at the same time, so the occurrence and spread of cascading failures are based on the network coupled with "topological structure" and "flow distribution structure." Therefore, when the passenger flow is loaded on a topological PTN as the weight, it is significant to study the cascading failures and their dynamical behaviors in the weighted PTN which is represented by the coupling function between "topological structure" and "flow distribution structure" in different strength.

Here, we propose a model to describe the cascading failures in weighted networks based on a CML model presented by Wang and $\mathrm{Xu}[43,44]$. The model is demonstrated as follows:

$$
\begin{aligned}
x_{i}(t+1)=\mid\left(1-\varepsilon_{1}-\varepsilon_{2}\right) f\left(x_{i}(t)\right)+\varepsilon_{1} \sum_{j=1, j \neq i}^{N} \frac{a_{i j} f\left(x_{j}(t)\right)}{k_{i}} \\
+\varepsilon_{2} \sum_{j=1, j \neq i}^{N} \frac{w_{i j} f\left(x_{j}(t)\right)}{s_{i}} \mid, \quad i=1,2, \ldots, N,
\end{aligned}
$$

where $x_{i}(t)$ is a state variable of the $i$ th node at the $t$ th time step. The connection information among $N$ nodes is given by the adjacency matrix $A=\left(a_{i j}\right)_{N \times N}$. If there is an edge between node $i$ and node $j, a_{i j}=a_{j i}=1$; otherwise, $a_{i j}=a_{j i}=0$. $k_{i}$ denotes the degree of node $i$; $w_{i j}$ represents the weight of edge between node $i$ and node $j ; s_{i}$ is the strength of node $i$. $\varepsilon_{1}$ means the coupling strength of topological structure and $\varepsilon_{2}$ represents that of weighted network which is loaded by passenger flow, respectively, where $\varepsilon_{1}, \varepsilon_{2} \in(0,1), \varepsilon_{1}+\varepsilon_{2}<1$. The function $f$ demonstrates the local dynamic behaviors which is chosen in this work as the chaotic logistic map, $f(x)=4 x(1-x)$. When $0 \leq x \leq 1$ and $0 \leq f(x) \leq 1$, we use absolute value notation in (2) to guarantee that the state of each node is always nonnegative.

Here, the nonlinear function $f$ can be defined as the evolution rule of node $i$ 's capacity. According to the mapping method of L-space, node $i$ is said to be in a normal state and its flow is smaller than its capacity at the $l$ th time step if $0<x_{i}(t)<1, t \leq l$. In contrast, if $x_{i}(l) \geq 1$, it means that the flow is overloaded on node $i$ at the $l$ th time step and then node $i$ is said to be failed, and we assume in this case that $x_{i}(t) \equiv 0, t>l$. The state of a node evolves in accordance with the function, if the initial states of all $N$ nodes in the network are in the interval $(0,1)$ and there is not any external perturbation or the flow surges, then all nodes in the network will be in a normal state forever, and the PTN will operate normally.

3.2. Methods for Analysis of Dynamic Robustness Based on CML Model. In order to show how an initial shock at a

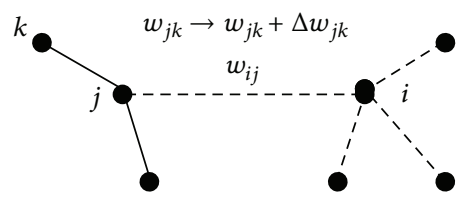

FIGURE 4: The adjustment rule of $w_{i j}$ after node $i$ is failed.

single node triggers cascading failures, we add an external perturbation $R \geq 1$ to node $i$ at the $m$ th time step as follows:

$$
\begin{array}{r}
x_{i}(m)=R+\mid\left(1-\varepsilon_{1}-\varepsilon_{2}\right) f\left(x_{i}(m-1)\right)+\varepsilon_{1} \\
+\varepsilon_{1} \sum_{j=1, j \neq i}^{N} \frac{a_{i j} f\left(x_{j}(m-1)\right)}{k_{i}} \\
+\varepsilon_{2} \sum_{j=1, j \neq i}^{N} \frac{w_{i j} f\left(x_{j}(m-1)\right)}{s_{i}} \mid .
\end{array}
$$

In this case, node $i$ will be failed at the $m$ th time step; namely, stop $i$ of a PTN is out of operation due to natural events or other emergencies and then the topological structure will change accordingly and the passenger flow will be redistributed. Therefore, we have $x_{i}(t) \equiv 0$ for all $t>m$. At the $(m+1)$ th time step, the states of those nodes directly connected with node $i$ will be affected by $x_{i}(m)$ according to (2), and their states may also be larger than 1 and thus may lead to a new round of nodes' failures. Nodes' failures will lead to a redistribution of the flow and spread out with a continued process.

The rule of flow redistribution is like this: when node $i$ failed, node $i$ and the edges connected with it will be excluded from the network, as shown in Figure 4 , and $w_{i j}$, the weight of edge linked with node $j$ and the failed node $i$, will be shared by other edges connected with node $j$, so their original weights will be changed: $w_{j k} \rightarrow w_{j k}+\Delta w_{j k}$; these edges share the flow according to their weights: $\Delta w_{j k}=w_{i j} * w_{j k} / s_{j}$. Generally, this rule exists in real PTNs because passengers would be much more likely to choose the nearby transit services to escape from the confusion and accident as soon as possible when a stop or route suffers from disaster or attack.

During the spreading process of cascading failures, the problem needed to be studied is as follows: what is the relationship between the scale of cascading failures and the strength of external perturbation in real weighted PTNs? Is there any threshold to avoid the cascading failures? What is the relationship between the spread of cascading failures and different coupling strength of topology and flow distribution structures under different attacks? And what are the similarities and dissimilarities of the cascading failures in different PTN structures?

Before following simulations, the initial states of nodes are all chosen randomly from the interval $(0,1)$. A perturbation with $R \geq 1$ is added to a node $c$. $I(t)$ is defined as the total number of failed nodes in the network before the $t$ th time step, and failure proportion is the ratio of $I(t)$ to 
the network's scale $N$. The failed nodes and failed edges are excluded from the network and flow is redistributed in terms of rule of Figure 4; then the spreading process of failures can be observed in the network. When there are no failures, $I$ is defined as the scale of the failures in the network, $I \equiv$ $\lim _{t \rightarrow \infty} I(t)$.

\section{Analysis of Cascading Failures in Beijing's Weighted BSN}

In Sections 4 and 5, we will take Beijing's BSN and MPTN systems as cases, with consideration of modes of random fault and intentional attack, to analyze their tolerance and robustness with the above CMLs model for weighted network. In the following mode of random fault, all simulation results are averages over 50 random realizations.

\subsection{Threshold for Cascading Failure}

4.1.1. When a Randomly Chosen Node Fails. Here, under random fault, the thresholds to trigger global cascading failures in Beijing's BSN are studied with and without flow redistribution, respectively, based on our CMLs model. Given $\varepsilon_{1}=\varepsilon_{2}=0.25$, a perturbation is added to a randomly selected node. As shown in Figure 5(a), considering flow redistribution based on the rule shown in Figure 4, when $R>R_{B 1}^{R}=3$, failures start to be triggered in the network; however, when not considering flow redistribution, the threshold $R_{B 2}^{R}=2$ becomes smaller (Figure 5(b)). It reflects that the passengers' travel behaviors with autonomy and flexibility can reduce the risk of cascade failure in the BSN. At the same time, we find that since the topology of Beijing's BSN is small-world and the weighted one is scalefree, the thresholds $R_{B 1}^{R}, R_{B 2}^{R}$ are both relatively small, which is in accordance with the results of $[43,44]$ that cascading failures are much easier to occur in small-world and scalefree topological CMLs than in global CMLs.

As shown in Figure 6, we find that there is a normal distribution relation between the scale and time step of cascading failures. When considering flow redistribution (Figure 6(a)), no matter how much $R$ changes, the failure is triggered earlier, peak times are concentrated in a smaller range $15<t_{p 1}<25$, and nodes are all failed in an earlier time step $t_{1}^{R}(e)=80$, comparing with that without flow redistribution (Figure $6(\mathrm{~b})$ ). It implies that the flow redistribution has the potential to enhance the ability of synchronization in bus transit system.

4.1.2. When a Specified Node Is Intentionally Attacked. Here, an intentional perturbation is added to nodes with the largest degree or strength, respectively; namely, the most important stations are intentionally attacked in the BSN, which are Guanganmennei station with $k=8$ and Military Museum station with $s=60918$. Similarly, we set $\varepsilon_{1}=\varepsilon_{2}=0.25$. When the stop with the largest degree is attacked (Figure 7(a)), considering flow redistribution, the threshold of failure is $R_{B}^{K}=2$, which is smaller than that of the stop with the largest strength, $R_{B}^{S}=3$ (Figure $8(\mathrm{a})$ ), indicating that the topology is more vulnerable than the flow distribution structure. The threshold is less than or equal to that of random fault; namely, $R_{B}^{K}<R_{B 1}^{R}$, but $R_{B}^{S}=R_{B 1}^{R}$, revealing that topological structure is more vulnerable against intentional attack, but that of the flow distribution structure surprisingly keeps the same robustness against random fault or intentional attack. We also find that the thresholds of degree-based and strengthbased attacks are the same with those of the case without considering flow redistribution, respectively (Figures $7(\mathrm{~b})$ and $8(\mathrm{~b})$ ), which reflects that when the most central nodes in the network are intentionally attacked, though passengers can change their travel routes, the flow changes in a small scope are not sufficient to reduce the impact of failures because the damage is very huge and failures spread much faster.

The further analysis of Figure 7(a) presents that when the degree-based attack occurs and $R>R_{B}^{K}$, there is a diversity for the failure proportion distribution and peak time with the change of $R$. When the perturbation is small, that is, $R=3,4$, the failures do not spread very quickly at first until $t=20$ and there is a turning point that the failures increase sharply, before which there could be a good opportunity to control failure to spread further; but when $R \geq 5$, it seems that the spreading processes are synchronized with different $R$, and the peak proportions are all $p_{k} \approx 0.07$ at a similar peak time $t_{p k}=12$. However, when the strength-based attack triggers failures (Figure 8(a)), the spreading speeds are similar for different $R$ values, and the peak proportions are $p_{s} \in(0.06,0.07)$ in a quite small time interval $t_{p_{s}} \in[12,18]$, indicating that once failure is triggered, the perturbation strength has little effect on the failure spreading process for the weighted BSN.

4.2. Effect of Flow Coupling Coefficient $\varepsilon_{2}$. The following will study the impacts of different flow coupling coefficients $\varepsilon_{2}$ on the robustness of Beijing's BSN. Firstly, a perturbation $R=5>R_{B 1}^{R}$ is added to a randomly selected node with $\varepsilon_{1}=0.25$ and $\varepsilon_{2}$ taking the values of $0.15,0.25,0.35,0.45$, and 0.55 , respectively. Both Figures 9 (a) and 9 (b) show that, on the whole, with $\varepsilon_{2}$ increasing, the failures occur earlier and spread faster with and without flow redistribution. However, when considering passengers change their travel routes, $\varepsilon_{2}$ has less influence on spreading processes of failures (shown in Figure 9(a)) comparing with that without flow redistribution (shown in Figure 9(b)), and there is less diversity between the initial occurrence times or spreading speeds of failures with different $\varepsilon_{2}$. In addition, as shown in Figure 9(a), the failures spread faster. These characteristics imply that, because of the flow redistribution in network, it is equivalent to increasing the coupling strength further between other stops and then promoting the spread of failure. It is important to note that when $\varepsilon_{2} \leq \varepsilon_{1}$, the peak proportion with flow redistribution is smaller than that of without it, indicating that flexibility of passengers' travel behaviors will be likely to alleviate the damage of perturbation under a small flow coupling strength.

For the case of intentional attack, with the same parameter set as the above, a perturbation $R=5>R_{B}^{K}$ and $R_{B}^{S}$ is added to the node with the largest degree or strength, 


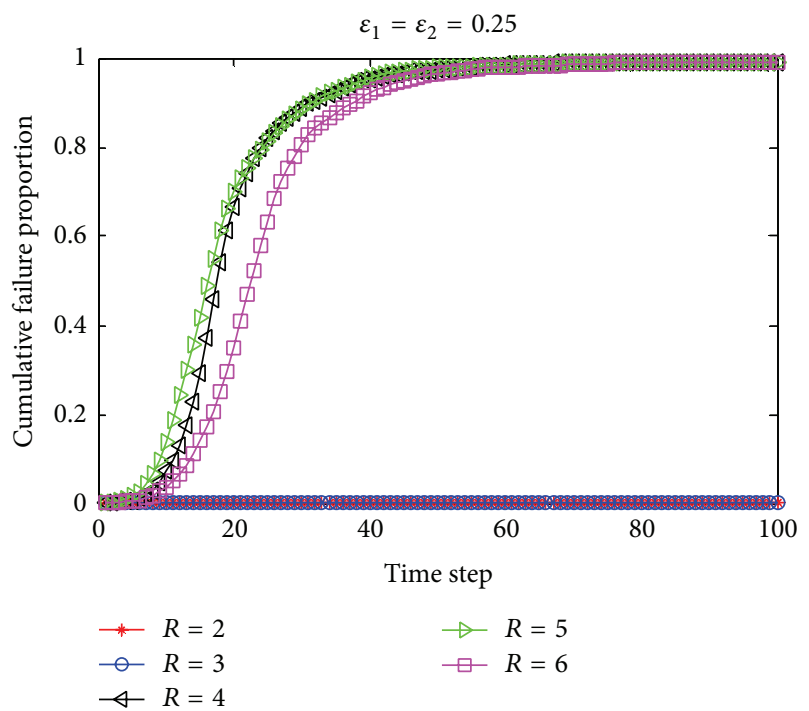

(a) Considering flow redistribution

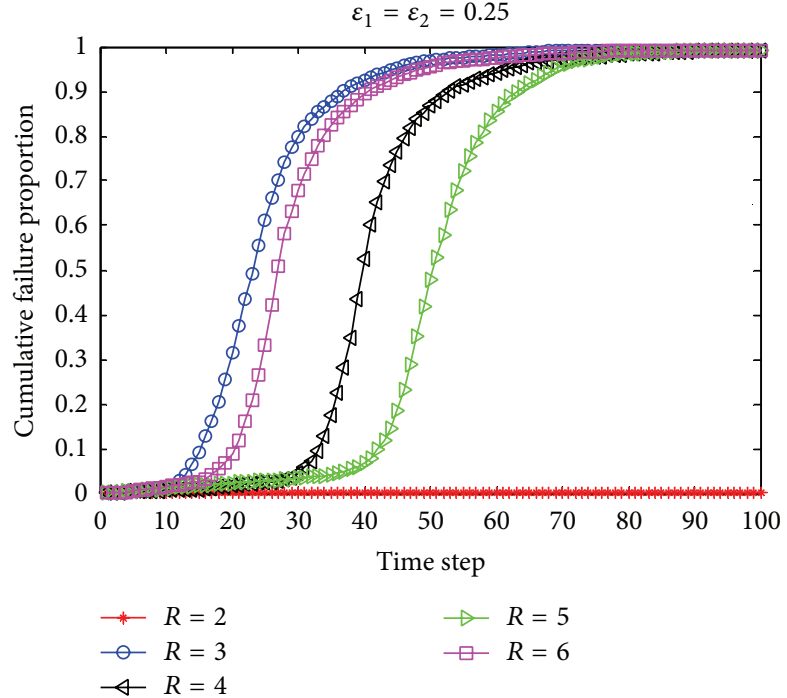

(b) Without considering flow redistribution

FIGURE 5: When $\varepsilon_{1}=\varepsilon_{2}$, a randomly chosen node fails, the spreading process of cascading failure with different perturbations $R$ in Beijing's weighted BSN.

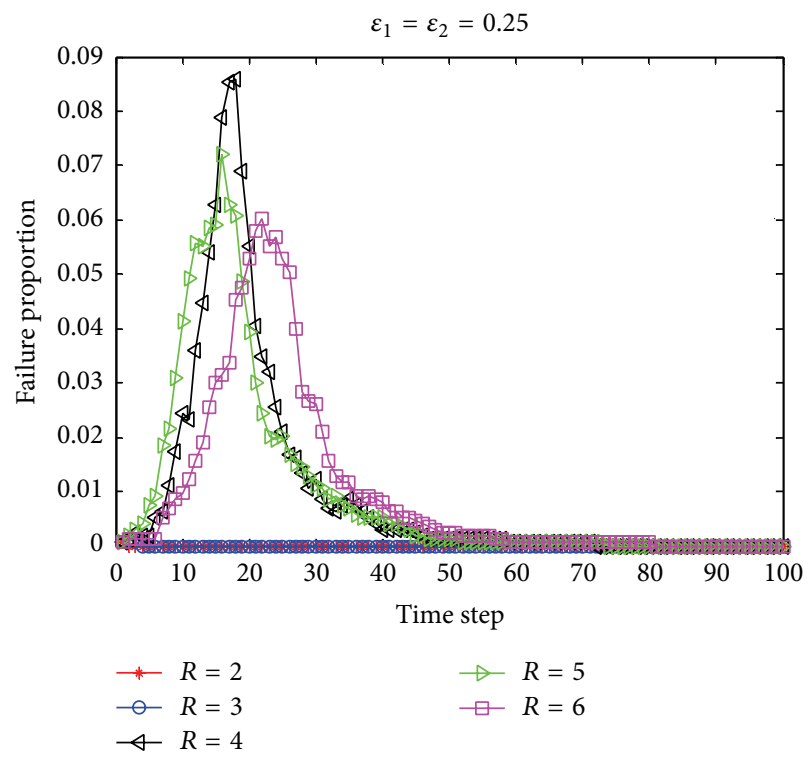

(a) Considering flow redistribution

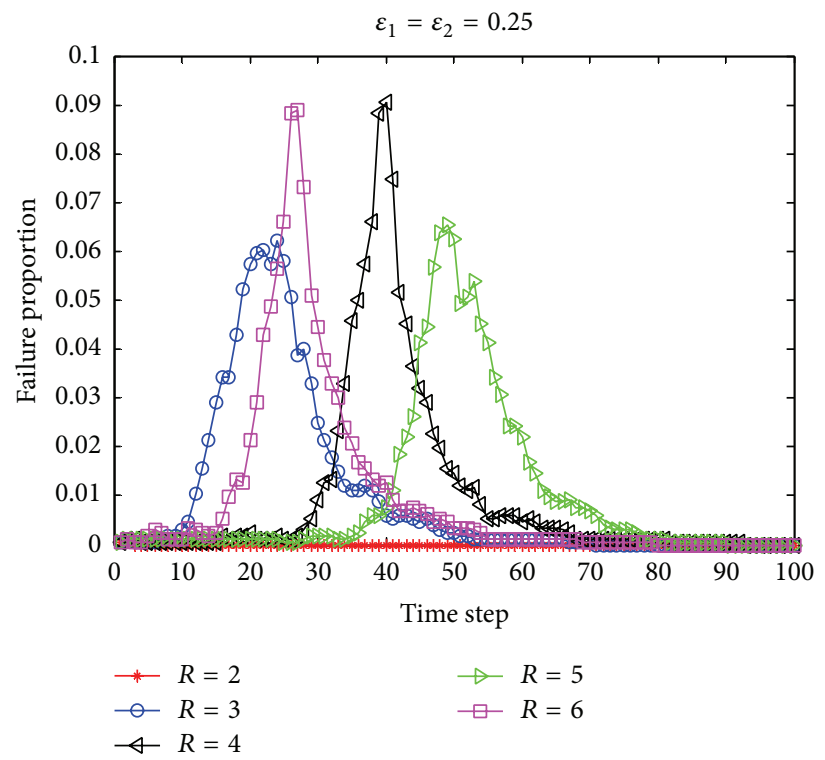

(b) Without considering flow redistribution

FIGURE 6: When $\varepsilon_{1}=\varepsilon_{2}$, a randomly chosen node fails, the relationship between failure scale and time step with different perturbations $R$ in Beijing's weighted BSN.

respectively. The simulations (shown in Figure 10) show that whether the stop with the largest degree or strength is intentionally attacked, the spreading processes of failures are almost synchronized for different $\varepsilon_{2}$; that is, the peak times all occur in a small range $t_{p_{k}} \approx t_{p_{s}} \in[12,16]$, and their failure proportions are $p_{k} \in[0.07,0.08]$ and $p_{s} \in[0.06,0.07]$, respectively, which reflects that although the topological network is small-world and the flow distribution is scalefree, the flow dynamic behaviors may nearly eliminate the cascading difference between these two structures. The time step that all of nodes are failed is $t_{k}(e)=74$ when the largest degree stop is attacked, which is later than that of the largest strength one; that is, $t_{s}(e)=69$, but they are both earlier than that in the mode of random fault $t(e)=82$, revealing that the spreading process is the most intense when the largest strength node is intentionally attacked.

4.3. Effect of Topological Coupling Coefficient $\varepsilon_{1}$. Here, we study the impacts of different topological coupling coefficients $\varepsilon_{1}$ on the network. Assume that $R=5, \varepsilon_{2}=0.25$ and $\varepsilon_{1}$ 


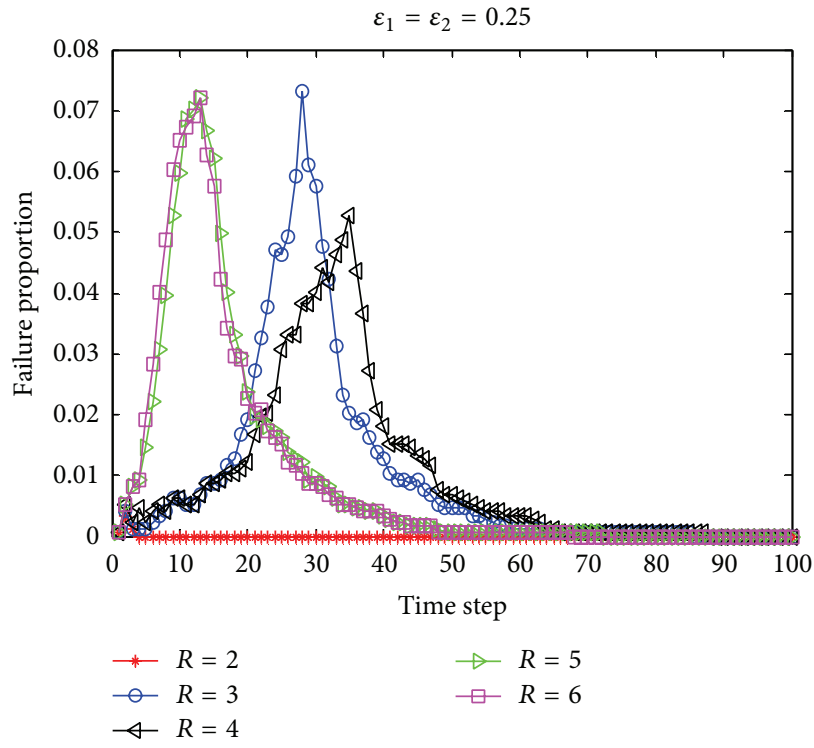

(a) Considering flow redistribution

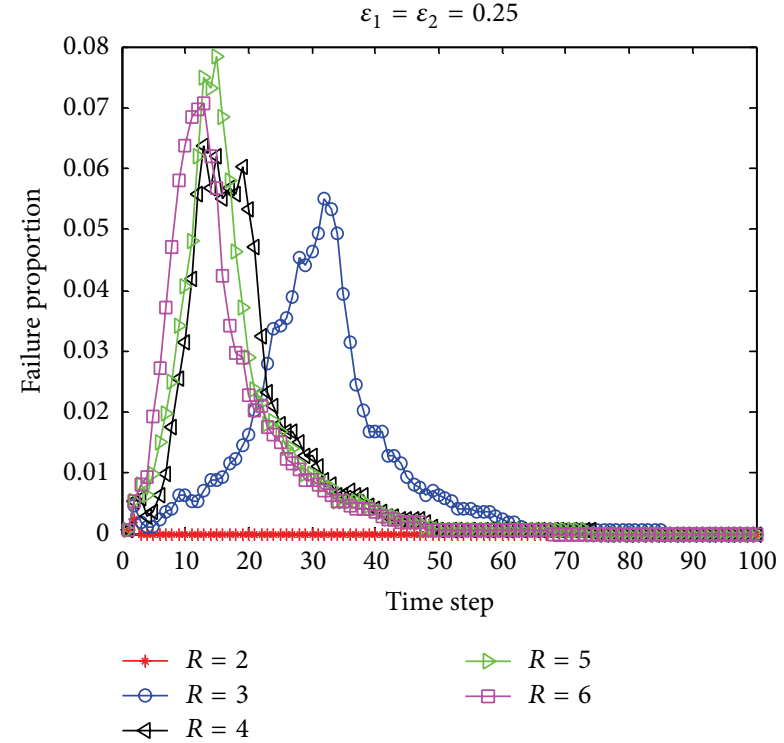

(b) Without considering flow redistribution

FIGURE 7: When $\varepsilon_{1}=\varepsilon_{2}$, the node with the largest degree is intentionally attacked, the relationship between failure scale and time step with different perturbations $R$ in Beijing's weighted BSN.

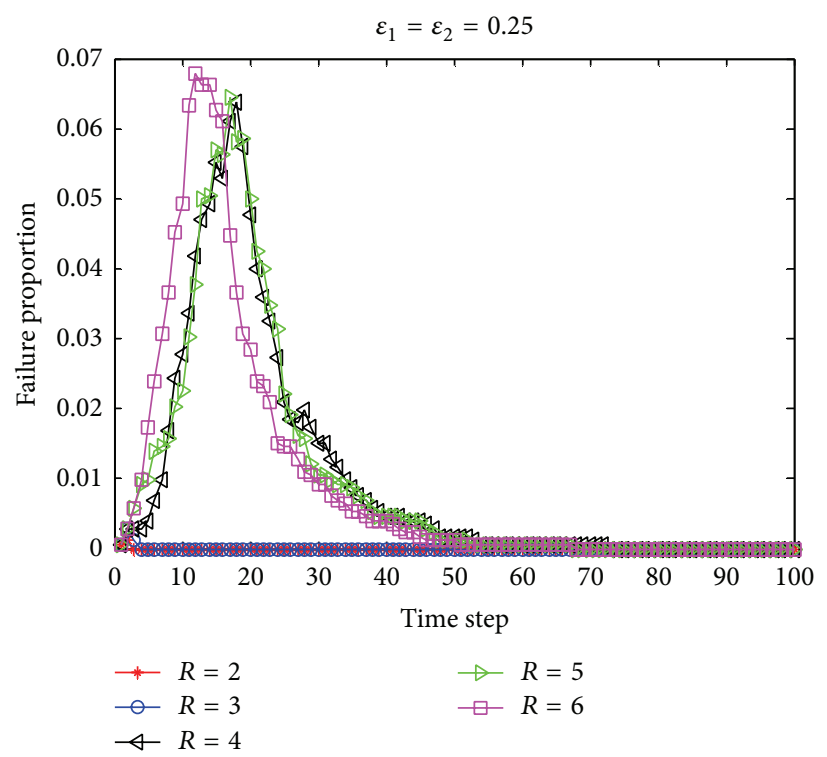

(a) Considering flow redistribution

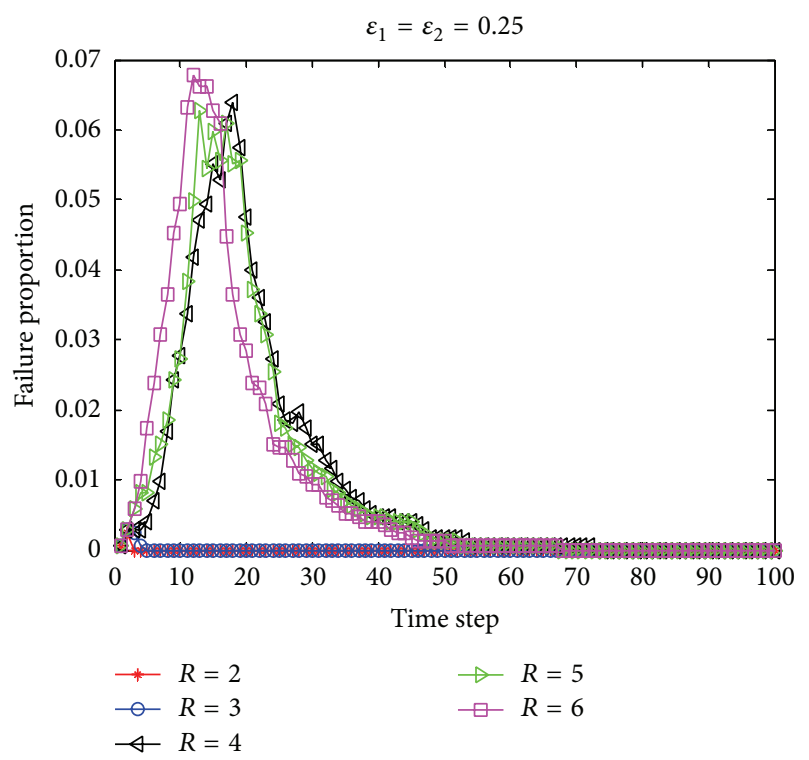

(b) Without considering flow redistribution

FIGURE 8: When $\varepsilon_{1}=\varepsilon_{2}$, the node with the largest strength is intentionally attacked, the relationship between failure scale and time step with different perturbations $R$ in Beijing's weighted BSN.

takes the values of $0.15,0.25,0.35,0.45$, and 0.55 , respectively. When a perturbation is added to a randomly selected node, according to Figure 11, we find that when considering flow redistribution, the spreading process of failures is much consistent and less diverse compared with that without flow redistribution, and both the failure peak times $t_{p_{r}} \in[18,20]$ and proportions $p_{r} \in[0.07,0.09]$ occur in a small range. It shows that, in random fault mode, because of the coupling function by flow redistribution, the impact of topological coupling strength on the spreading process of failure has been mitigated.

When the node with the largest degree or strength is intentionally attacked, as shown in Figure 12, if $\varepsilon_{1}<\varepsilon_{2}$, the failures spread more slowly correspondingly, and there are some fluctuations for failure proportion at certain time steps, unlike other cases which failures always increase before the peak; and the time $t_{p_{k}}=33$ that reaches the failure peak under the degree-based attack (Figure 12(a)) is later than that 


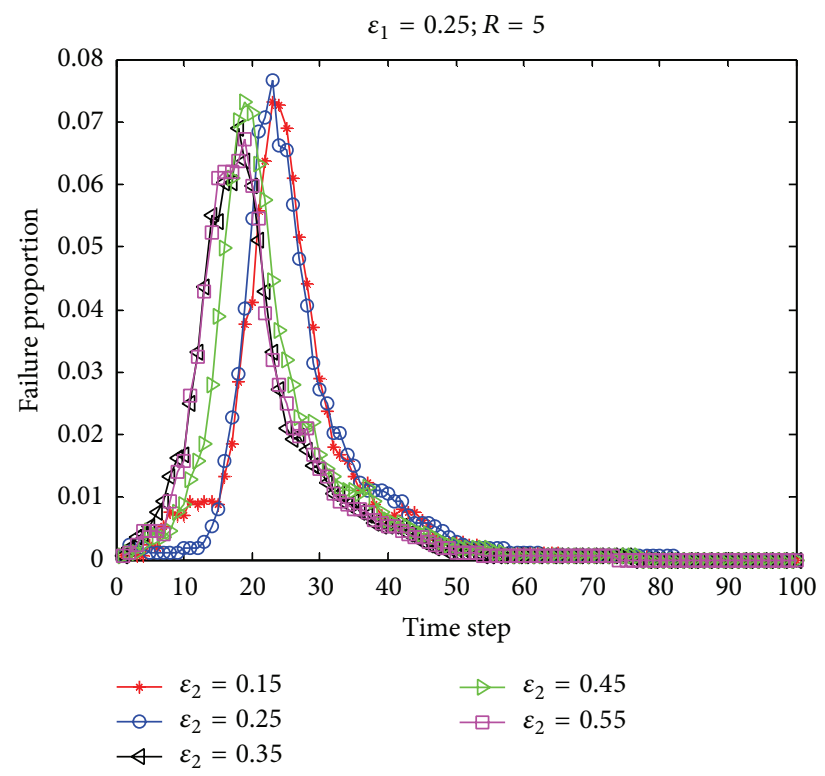

(a) Considering flow redistribution

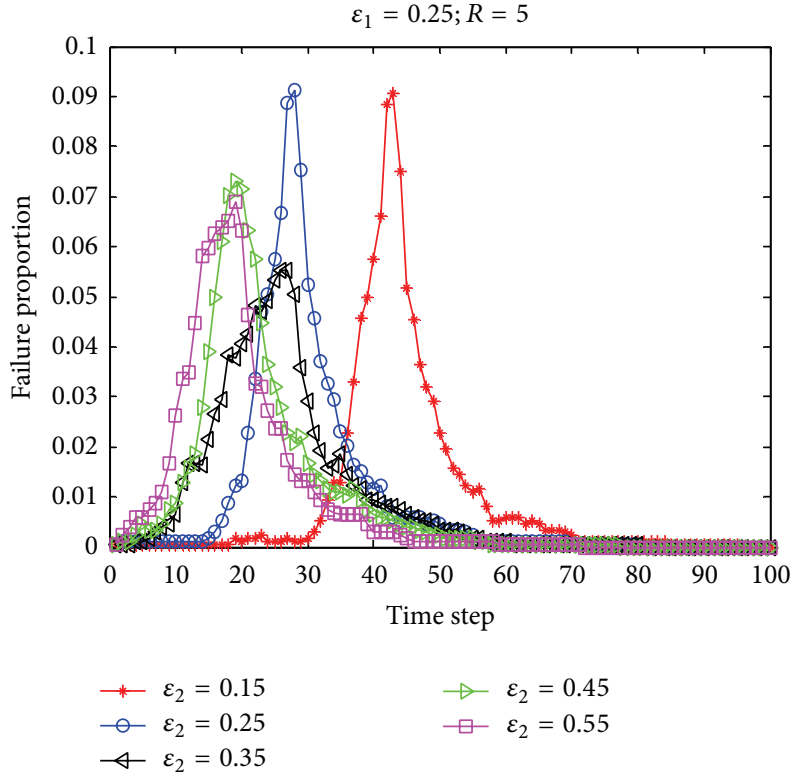

(b) Without considering flow redistribution

FIGURE 9: When a randomly chosen node fails, the relationship between failure scale and time step with different flow coupling coefficients $\varepsilon_{2}$ in Beijing's weighted BSN.

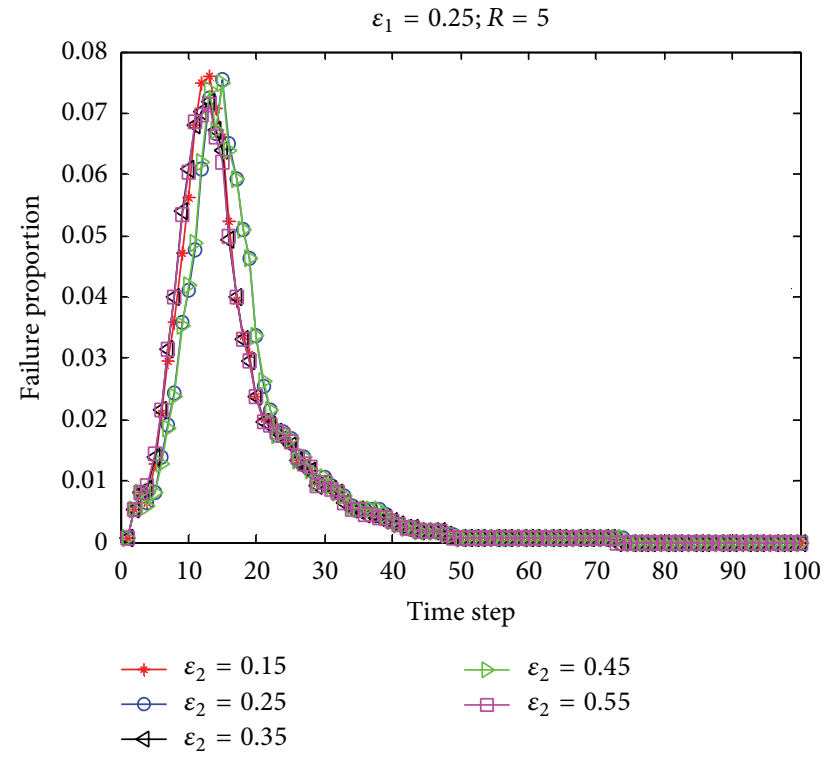

(a) Node with the largest degree is intentionally attacked

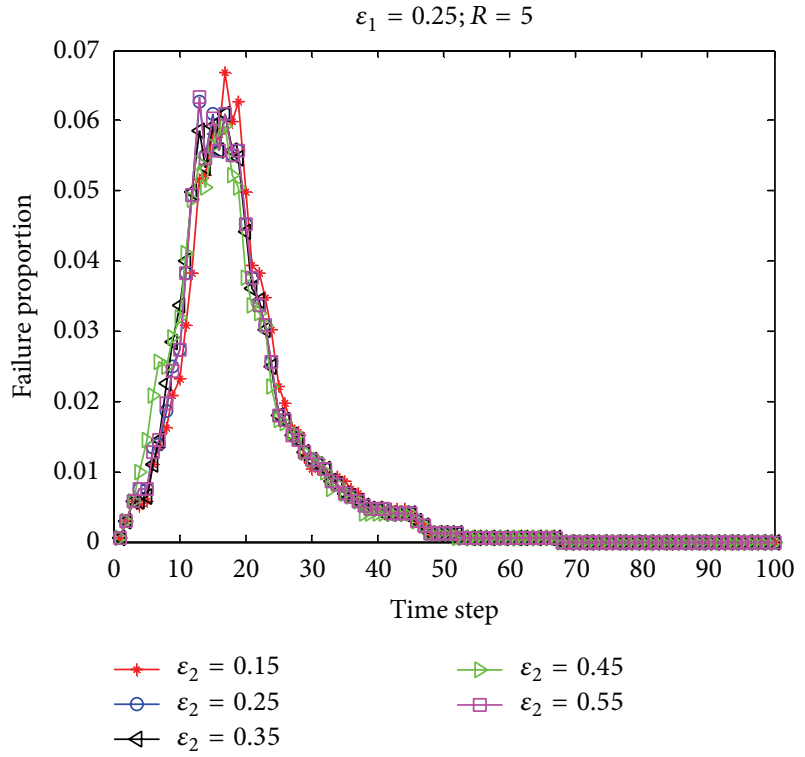

(b) Node with the largest strength is intentionally attacked

FIGURE 10: When a specified node is intentionally attacked, considering flow redistribution, the relationship between failure scale and time step with different flow coupling coefficients $\varepsilon_{2}$ in Beijing's weighted BSN.

of strength-based attack, $t_{p_{s}}=22$ (Figure 12(b)). However, when $\varepsilon_{1}>\varepsilon_{2}$ and $\varepsilon_{1}+\varepsilon_{2}>0.5$, the spreading processes are nearly consistent for these two attacks; that is, the peak times are $t_{p_{k}} \approx t_{p_{s}} \approx 15$ and the peak proportion for degreebased attack is $p_{k} \in[0.07,0.08]$ and for strength-based is $p_{s} \in[0.06,0.07]$; the times when all nodes fail are $t_{k}(e)=74$ and $t_{s}(e)=69$, respectively, which are also earlier than that of random fault mode. To sum up, when $\varepsilon_{1}<\varepsilon_{2}$, intentional attack to the most central node in weighted BSN (strengthbased attack) will trigger a more intense spreading process of failure than that in the topology (degree-based attack); but when $\varepsilon_{1}>\varepsilon_{2}$, hardly does $\varepsilon_{1}$ influence the failure occurrence time, spreading speed, and scale, implying that, in this case, to optimize the flow distribution structure, for example, by making it more homogenous or evacuating the passengers in the most central stop, maybe is much more significant 


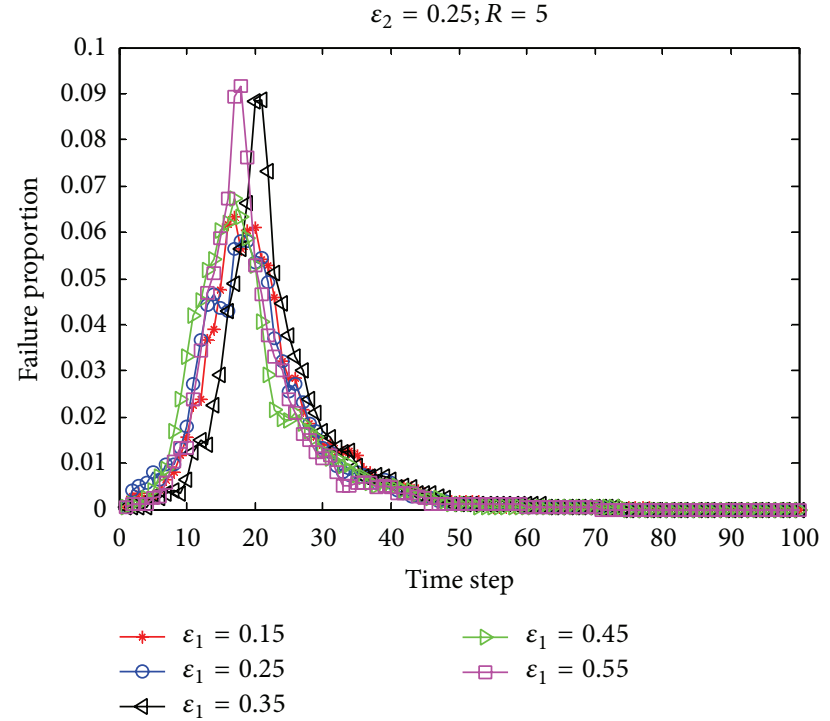

(a) Considering flow redistribution

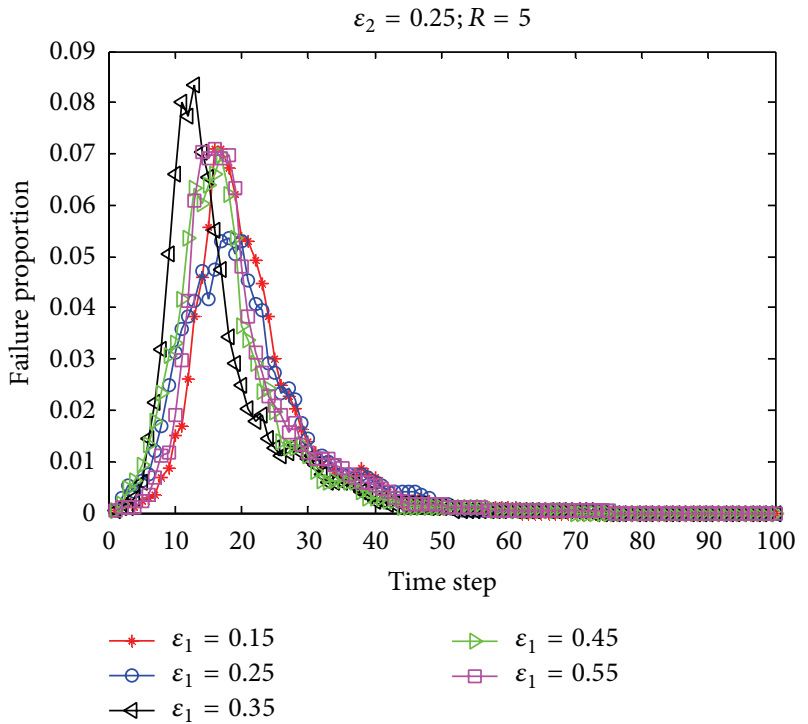

(b) Without considering flow redistribution

FIGURE 11: When a randomly chosen node fails, the relationship between failure scale and time step with different topological coupling coefficients $\varepsilon_{1}$ in Beijing's weighted BSN.

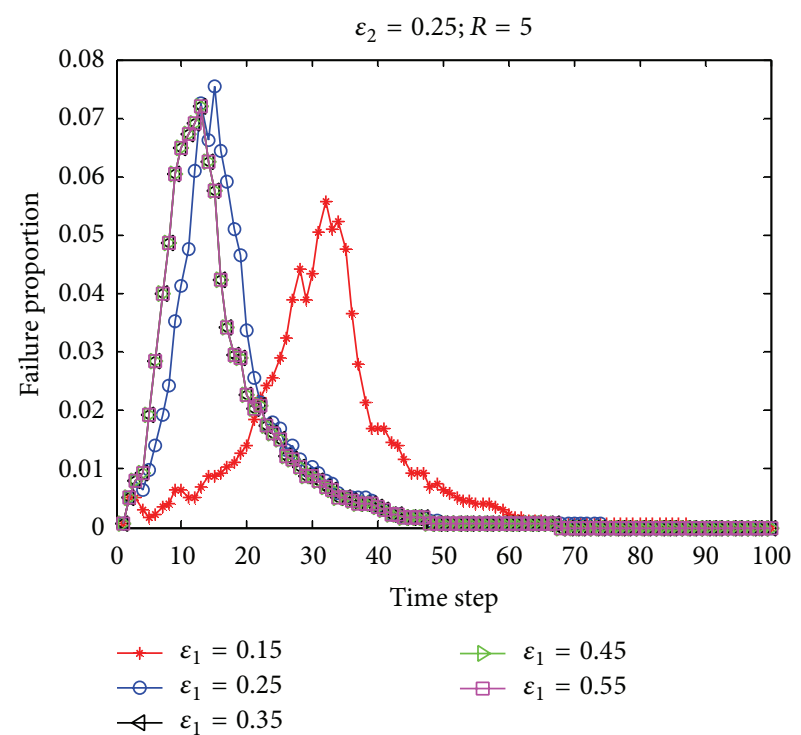

(a) Node with the largest degree is intentionally attacked

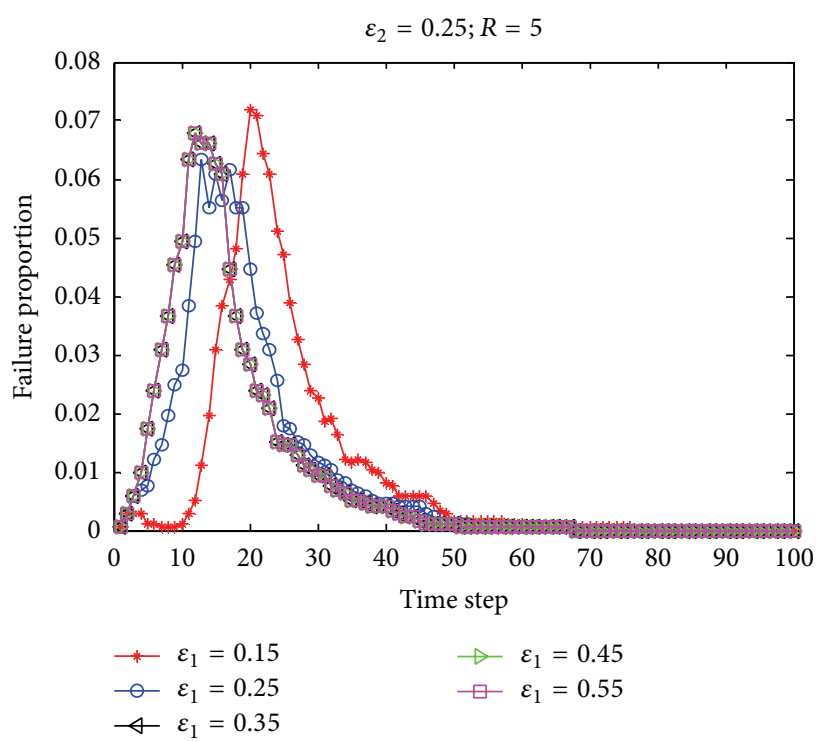

(b) Node with the largest strength is intentionally attacked

FIGURE 12: When a specified node is intentionally attacked, considering flow redistribution, the relationship between failure scale and time step with different topological coupling coefficients $\varepsilon_{1}$ in Beijing's weighted BSN.

since the topological coupling strength has little impact on the cascade while the flow redistribution can influence the spread of failure.

\section{Analysis of Cascading Failures in Beijing's Weighted MPTN}

5.1. Threshold for Cascading Failure. Here, the thresholds for cascading failures in MPTN are studied with consideration of flow redistribution, and the parameters are set the same as those in Section 4.1. As shown in Figure 13, in the random fault mode, when $R>R_{B+R}^{R}=2$, failures start to be triggered in the network, representing the fact that the threshold of MPTN $R_{B+R}^{R}=2$ is smaller than that of $\mathrm{BSN} R_{B 1}^{R}=3$. It reveals that the MPTN is more vulnerable than that of BSN under random fault, because both the topology and flow distribution of MPTN display much more heterogeneity according to our analysis in Sections 2.2 and 2.3, which is consistent with earlier works on cascading failures [21, 44], 


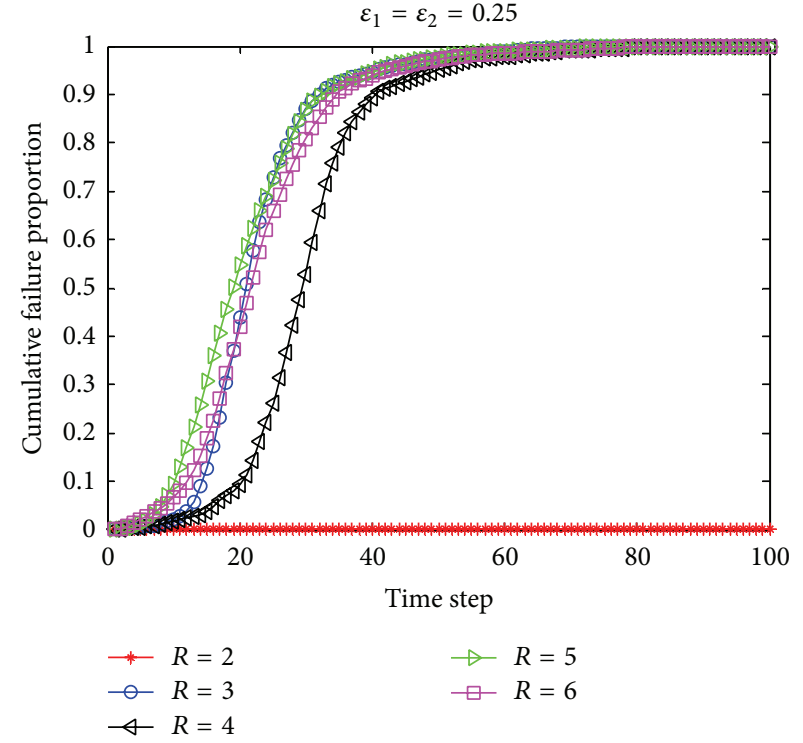

(a) Spreading process of failures

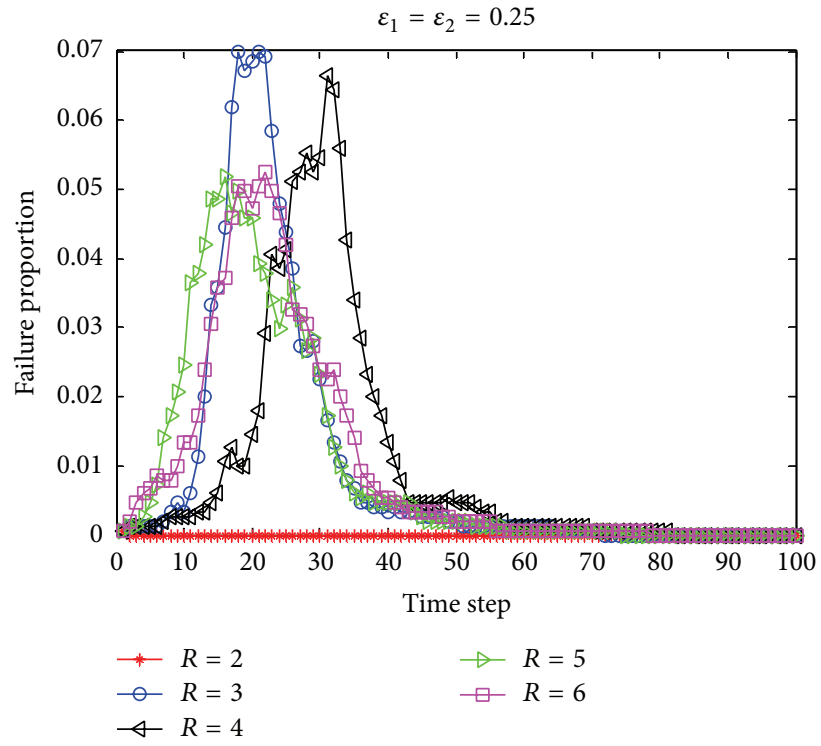

(b) Relationship between failure scale and time step

FIGURE 13: When $\varepsilon_{1}=\varepsilon_{2}$ and a randomly chosen node fails, considering flow redistribution, the threshold for cascading failure with different perturbations $R$ in Beijing's weighted MPTN.

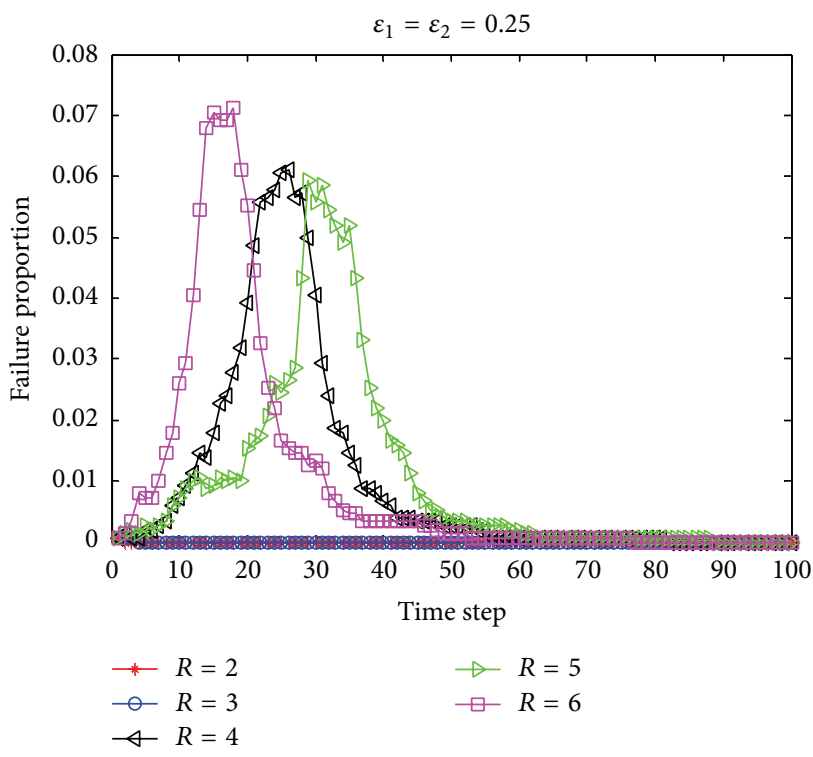

(a) Node with the largest degree is intentionally attacked

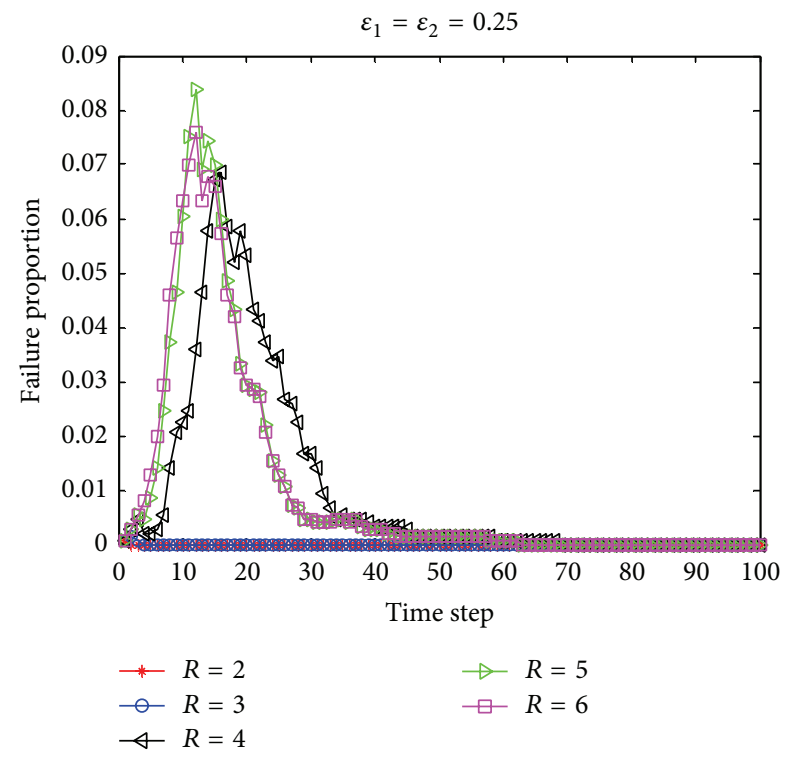

(b) Node with the largest strength is intentionally attacked

FIGURE 14: When $\varepsilon_{1}=\varepsilon_{2}$, a specified node is intentionally attacked, considering flow redistribution, the relationship between failure scale and time step with different perturbations $R$ in Beijing's weighted MPTN.

where it has been shown that large-scale cascading failures are much less likely to happen in a homogeneous network than in a heterogeneous network. In addition, as shown in Figure 13(b), when $R>R_{B+R}^{R}$, the initial occurrence time of failure becomes earlier while peak proportion could be smaller with the increase of $R$; for example, $R=4$ or 5 , indicating that the perturbation strength is not linear with the peak failure.
When the node with the largest degree or strength is attacked, respectively (Figure 14), that is, Sanyuanqiao station with $k=10$ and Jianguomen station with $s=1056343$, the thresholds of failures in MPTN are the same as $R_{B+R}^{K}=$ $R_{B+R}^{S}=3$. Also, they are the same with BSN under strengthbased attack $R_{B}^{S}=3$, larger than BSN under degree-based attack $R_{B}^{K}=2$, and even larger than itself under random fault $R_{B+R}^{R}=2$, which implies that, because of the flow 


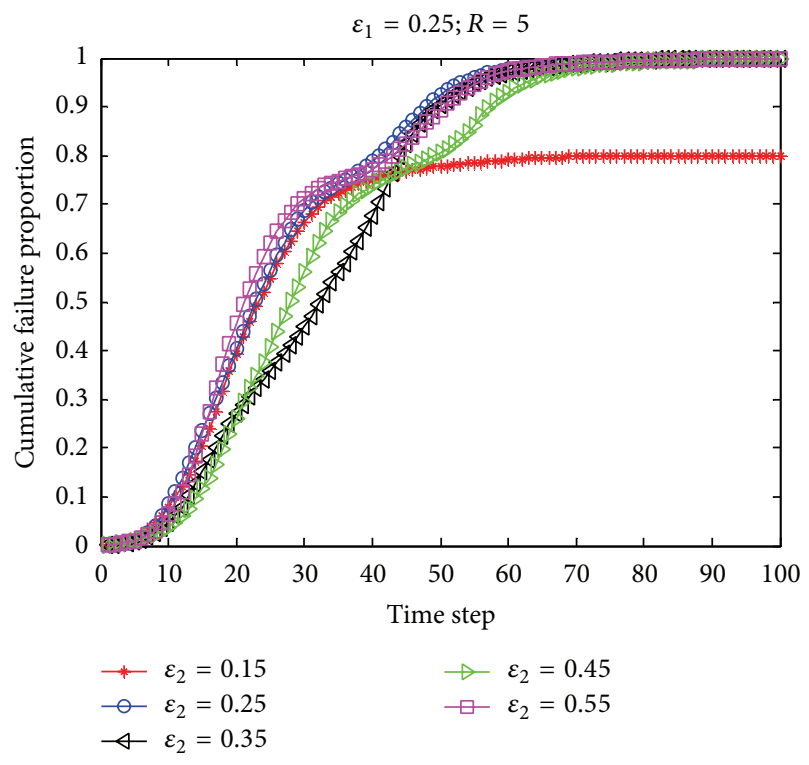

(a) Spreading process of failures

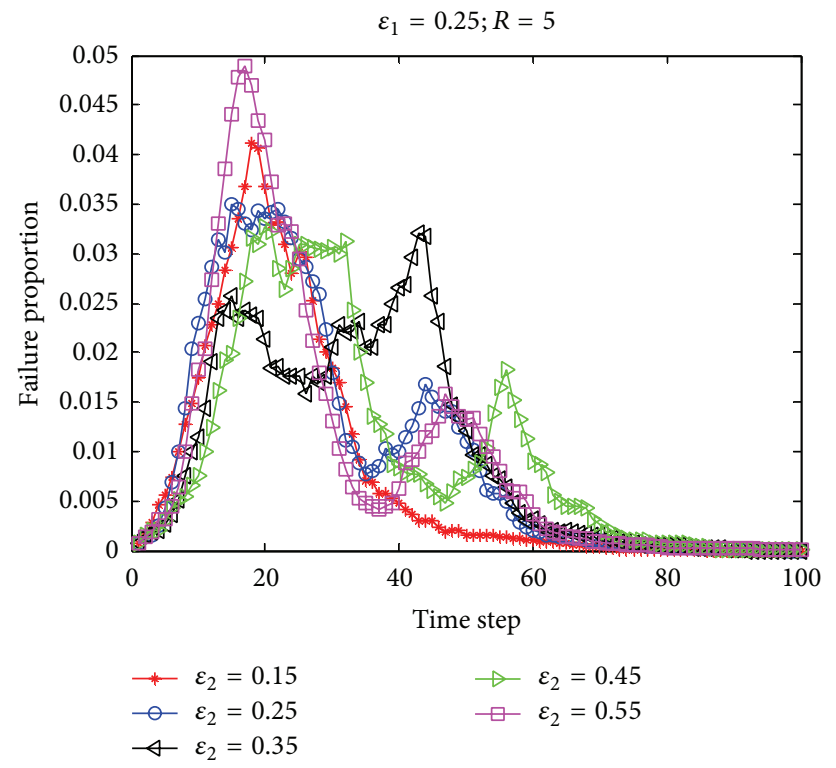

(b) Relationship between failure scale and time step

FIGURE 15: When a randomly chosen node fails, considering flow redistribution, the spreading process of cascading failure with different flow coupling coefficients $\varepsilon_{2}$ in Beijing's weighted MPTN.

redistribution and the coupling function with two transit modes of bus and rail, though the most important station is attacked, the ability of MPTN to resist perturbation can be surprisingly improved. At the same time, we find that, unlike BSN, since both topology and flow structures of MPTN are more heterogeneous, whether the node with the largest degree or strength is attacked, the initial failure is much more easily triggered with the increase of $R$, and failure spreads faster than that of random fault mode. It also shows that the spreading process under strength-based attack is much faster and more intense than that under degree-based attack, as the flow distribution of MPTN is much more heterogeneous than the topology.

5.2. Effect of Flow Coupling Coefficient $\varepsilon_{2}$. As shown in Figure 15, a perturbation $R=5$ is added to a randomly chosen node. When $\varepsilon_{2}=0.35$, failure spreads more slowly than those with other values of $\varepsilon_{2}$, and the peak comes later; its proportion $p_{B+R}^{R}\left(\varepsilon_{2}=0.35\right)=0.034$ is smaller or equal to those with other values of $\varepsilon_{2}\left(p_{B+R}^{R} \in[0.034,0.05]\right)$, indicating there could be an opportunity to control the cascading failure. We also find that, unlike BSN, there is no trend showing that $\varepsilon_{2}$ is linearly correlated with time step and failure proportion in the MPTN, and when $\varepsilon_{2}$ increases, the dynamic flow redistribution in the network even can delay the failure initial time and reduce the peak scale because of the heterogeneity of MPTN.

When the node with the largest degree is attacked (shown in Figure 16(a)), as $\varepsilon_{1}$ is being kept at the same value, there is a threshold $\varepsilon_{2}^{K^{*}}=0.4$, which means that when $\varepsilon_{2} \in(0,0.4]$ or $\varepsilon_{2} \in(0.4,1)$, no matter how much $\varepsilon_{2}$ changes, the spreading processes of failures present two synchronous curves, and the spreading processes with $\varepsilon_{2} \in(0,0.4]$ and those with $\varepsilon_{2} \in(0.4,1)$ are spectacularly consistent; at the same time, Figure 16(a) indicates that the failure initial times are earlier and the average peaks of failures are larger with $\varepsilon_{2} \in(0.4,1)$ than those with $\varepsilon_{2} \in(0,0.4]$.

This weak sensitivity of effect on cascading failure from $\varepsilon_{2}$ becomes more prominent when the largest strength node is attacked. As shown in Figure 16(b), the spreading processes are always the same and totally not affected by $\varepsilon_{2}$ values. According to the relationship between failure scale and time step, under strength-based attack, the cascading failure is much more intense than that under degree-based attack, and the failure peak occurs much earlier; that is, $t_{p_{s}}=12<t_{p_{k}}=$ 29 (when $\varepsilon_{2} \in(0,0.4]$ ) or 21 (when $\varepsilon_{2} \in(0.4,1)$ ), with higher peak value $p_{s}=0.086>p_{k}=0.06$ (when $\left.\varepsilon_{2} \in(0,0.4]\right)$ or 0.072 (when $\varepsilon_{2} \in(0.4,1)$ ); the time of network failed globally is also earlier: $t_{s}(e)=63<t_{k}(e)=78$, which is also earlier than the time $t(e)=92$ in random fault.

5.3. Effect of Topological Coupling Coefficient $\varepsilon_{1}$. In random fault mode, as shown in Figure 17, similar to BSN, with consideration of flow redistribution, $\varepsilon_{1}$ has less effect on the spreading processes of failures in MPTN with different values, and the peak times and peak proportions of failures appear in a small range; that is, $t_{B+R}^{R} \in[14,24]$ and $p_{B+R}^{R} \in$ $[0.062,0.078]$. Notably, as shown in Figure 17(b), when a large value of $\varepsilon_{1}$ is set as $\varepsilon_{1}=0.55$, the failure peak time is relatively late and its proportion is the smallest, indicating that the flow redistribution in a topology with high coupling strength may play a role to relieve the impact of cascading failure.

Figure 18 plots the spreading process of cascading failure when the node with the largest degree is intentionally attacked with different $\varepsilon_{1}$ and $\varepsilon_{2}=0.15$ or $\varepsilon_{2}=0.25$. We 


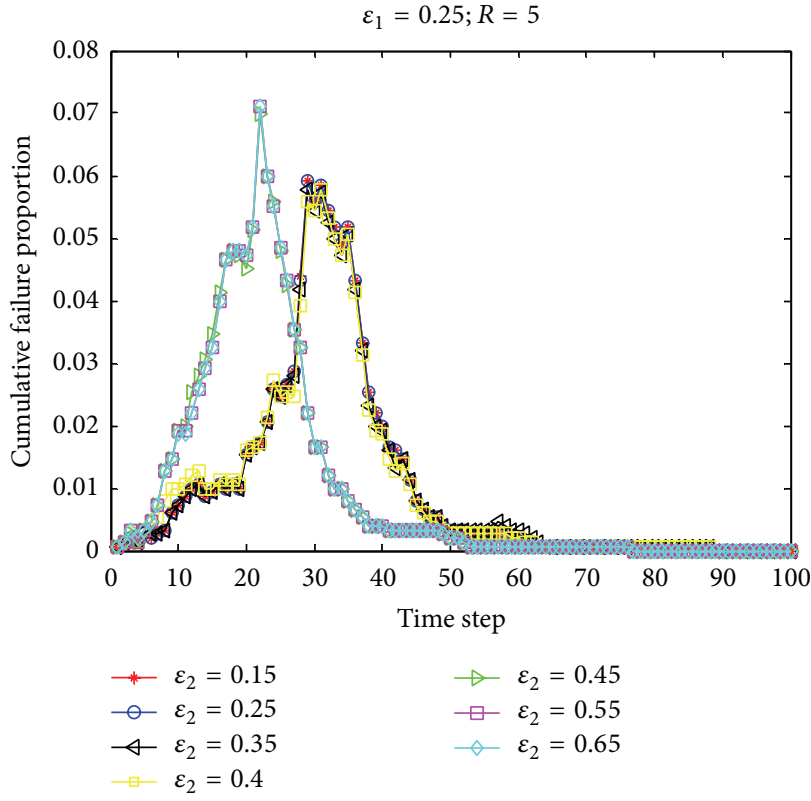

(a) Node with the largest degree is intentionally attacked

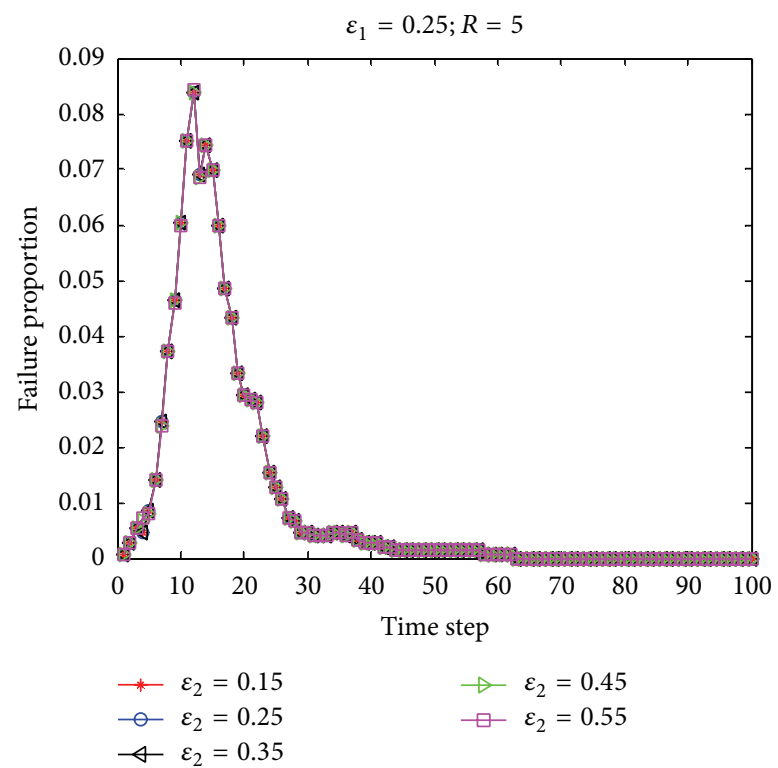

(b) Node with the largest strength is intentionally attacked

FIGURE 16: When a specified node is intentionally attacked, considering flow redistribution, the spreading process of cascading failures with different flow coupling coefficients $\varepsilon_{2}$ in the Beijing's weighted MPTN.

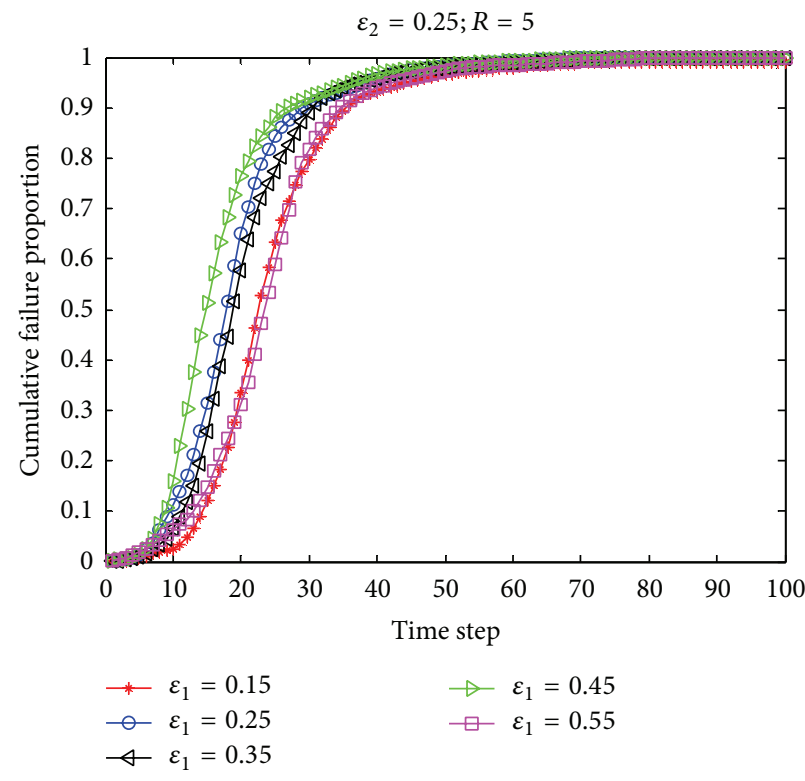

(a) Spreading process of failures

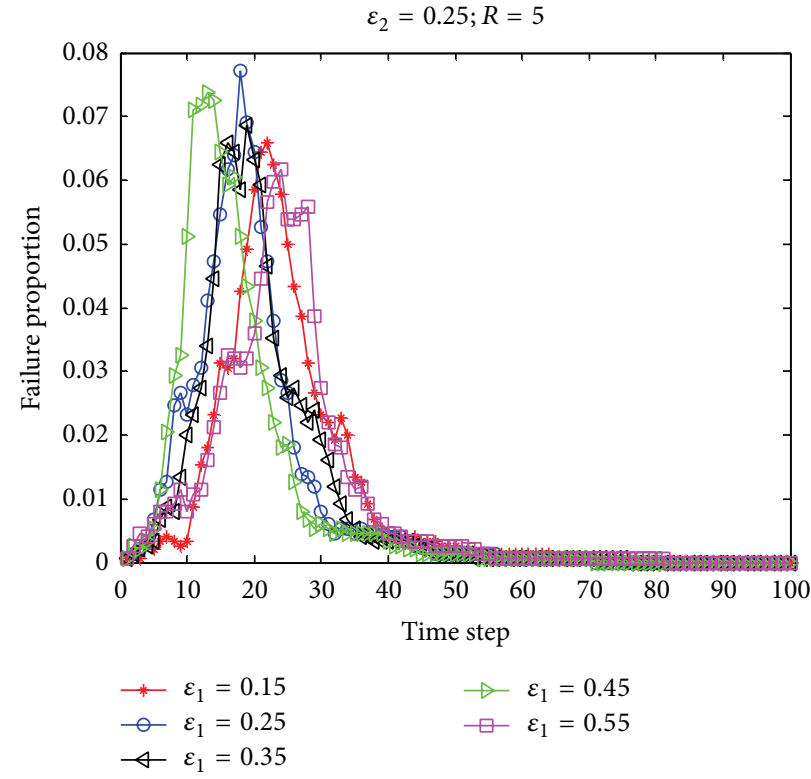

(b) Relationship between failure scale and time step

FIGURE 17: When a randomly chosen node fails, considering flow redistribution, the spreading process of cascading failure with different topological coupling coefficients $\varepsilon_{1}$ in Beijing's weighted MPTN.

find that there is a threshold of $\varepsilon_{1}^{K^{*}}=0.45$, namely, when $\varepsilon_{1} \geq$ 0.45 , whatever $\varepsilon_{1}$ increases, the spreading processes reach a synchronization; meanwhile, when $\varepsilon_{1} \geq 0.45$, the initial times of failures are earlier than those when $\varepsilon_{1}<0.45$, and the average peak proportion is also relatively larger. Likewise, under strength-based attack (shown in Figure 19), there is also a synchronization for spreading process with a threshold
$\varepsilon_{1}^{S^{*}}=0.25$ and when $\varepsilon_{1} \geq 0.25$, the initial and peak times of failures are earlier and the peak value is larger.

Similarly, we find that, no matter how topological coupling strength $\varepsilon_{1}$ changes, the spreading process of cascading failure is the most intense when the node with the largest strength is attacked; the threshold $\varepsilon_{1}^{S^{*}}=0.25$ in the case that the synchronization of cascade occurs is smaller than 


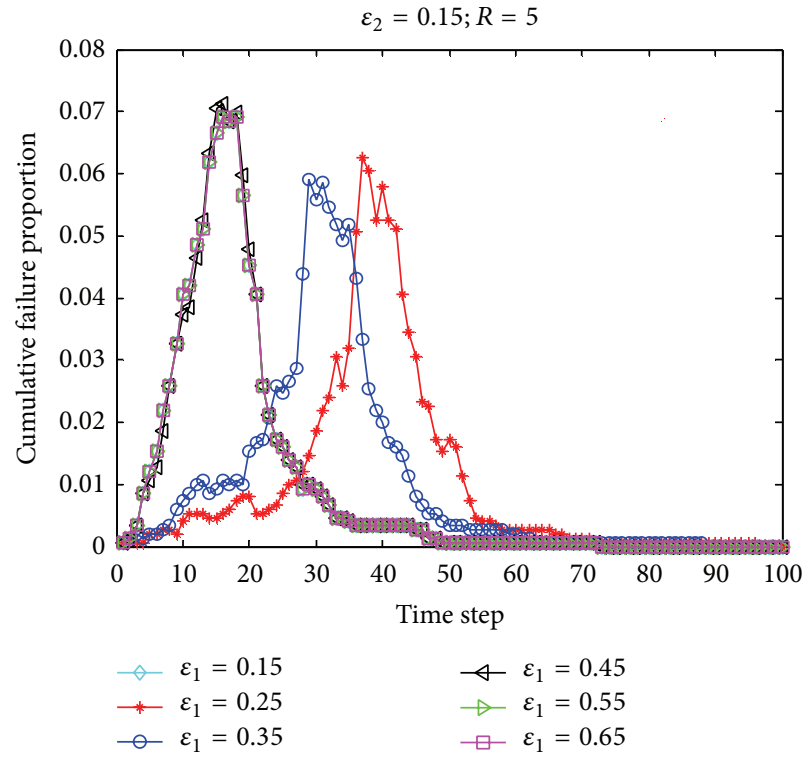

(a) When $\varepsilon_{2}=0.15$

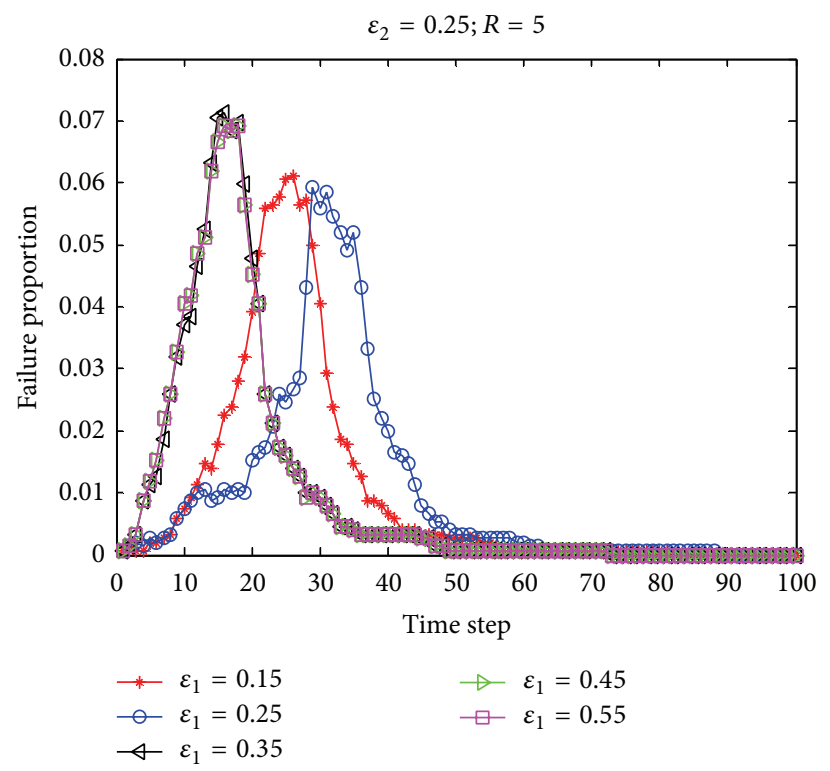

(b) When $\varepsilon_{2}=0.25$, relationship between failure scale and time step

FIGURE 18: When the node with the largest degree is intentionally attacked, considering flow redistribution, the spreading process of cascading failure with different topological coupling coefficients $\varepsilon_{1}$ in Beijing's weighted MPTN.

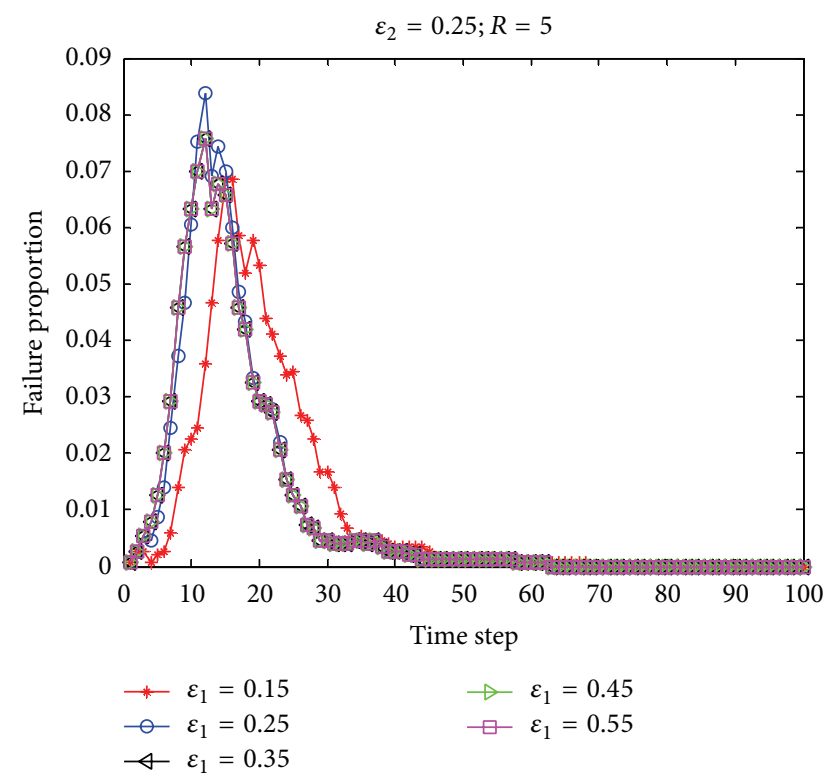

FIGURE 19: When the node with the largest strength is intentionally attacked, considering flow redistribution, the spreading process of cascading failures with different topological coupling coefficients $\varepsilon_{1}$ in the Beijing's weighted MPTN.

that of $\varepsilon_{1}^{K^{*}}=0.45$ when the largest degree one is attacked; after occurrence of synchronization, the peak time $t_{p_{s}}=12$ under strength-based attack is earlier than that of $t_{p_{k}}=17$ under degree-based attack, and the peak proportion is higher: $p_{s}=0.085>p_{k}=0.072$. It reveals that when the most important station in a weighted network is attacked, even though the topology is not coupled so much, the MPTN will suffer much heavier damage, as both of its topology and flow distribution structures are scale-free, which is consistent with the conclusions of $[2,43,44]$.

\section{Conclusions}

In summary, we find that both weighted BSN and MPTN in Beijing are scale-free networks while the topology of the former is small-world and that of the latter is scale-free, which make them show different robustness against different kind of faults or attacks. According to the thresholds for cascading failures, we find that when considering the dynamic flow behavior, the network displays a higher tolerance against random fault than that without flow redistribution. Particularly, in the weighted PTN based on passenger flow as a scalefree network, even the station with the largest strength is intentionally attacked; because of the dynamic flow redistribution, the network displays a surprisingly increasing degree of robustness compared with topological network with small-world structure against intentional attack, or even with scale-free one against random fault. This should encourage the development of multimodal transit networks in large metropolitan areas. It is the passengers' choice behaviors that play an important role to improve the robustness of PTN. It can explain why real PTNs rarely suffer complete breakdowns despite frequent disruptions from natural and man-made disasters.

However, once cascading failures are triggered in the network, we find that when the station with the largest strength is intentionally attacked, the damage to the network is much heavier than that of the largest degree-based attack, also, heavier than that of a randomly chosen node failed. This reminds us that we should pay more attention to the most 
central node (station), that is, the one with the largest strength in a weighted network to control the cascading failure, since the control window is short when cascading failure occurs. As the weighted PTN in Beijing is a scale-free network, these characteristics of vulnerability are consistent with the discoveries of Motter [21].

In essence, the flow redistribution has the ability to promote the synchronization during the spread of failure. Under intentional attack, although there is a certain degree of diversity for the spreading process with different topological coupling strength $\varepsilon_{1}$, some synchronizations tend to occur. However, the flow coupling strength $\varepsilon_{2}$ has little impact on the failure spread, especially for strength-based attack, and the synchronizations are much more likely to appear, indicating that when the most important station in the weighted PTN is attacked, the dynamic changes and redistribution of passenger flow can intensify the spreading process of cascading failure, no matter how much the initial flow is coupled. Fortunately, we can find some key thresholds of $\varepsilon_{1}$ and $\varepsilon_{2}$ that lead to synchronizations or make the cascading failure be triggered later or spread more slowly, which will help us find strategies to contain the spreading and avoid further damage.

The flow redistribution behavior adopted in this work is still primitive. More realistic behavior adaptations of major failures in transportation systems, such as the global redistribution of OD flows, should be explored. In addition, we only considered failures in nodes, while in the real world, failures in links also exist. When a route or route link that goes through a node fails, the node (and all connecting edges) does not necessarily have to be excluded from the network but only the failed links. Link failures in transportation system will be explored in our future study. Last but not least, further studies to better understand the complicated intrinsic properties of cascading failures and the essence of synchronization in weighted complex public transit networks are also worth pursuing.

\section{Conflict of Interests}

The authors declare that there is no conflict of interests regarding the publication of this paper.

\section{Acknowledgments}

This work is supported in part by the Fundamental Research Funds for the Central Universities of China (Approval no. 2014JBM071), the Major State Basic Research Development Program of China (973 Program) (Grant no. 2012CB7254035), the Key Program of National Natural Science Foundation of China (Grant no. 71131001-2), and China Scholarship Council (no. 201207095022).

\section{References}

[1] D. J. Watts and S. H. Strogatz, "Collective dynamics of "smallworld” networks," Nature, vol. 393, no. 6684, pp. 440-442, 1998.

[2] A. Barabási and R. Albert, "Emergence of scaling in random networks," Science, vol. 286, no. 5439, pp. 509-512, 1999.
[3] V. Latora and M. Marchiori, "Is the Boston subway a smallworld network?” Physica A, vol. 314, no. 1, pp. 109-113, 2002.

[4] K. A. Seaton and L. M. Hackett, "Stations, trains and smallworld networks," Physica A, vol. 339, no. 3-4, pp. 635-644, 2004.

[5] P. Sen, S. Dasgupta, A. Chatterjee, P. A. Sreeram, G. Mukherjee, and S. S. Manna, "Small-world properties of the Indian railway network," Physical Review E, vol. 67, no. 3, Article ID 036106, 5 pages, 2003.

[6] Y. Wang, T. Zhou, J. Shi, J. Wang, and D. He, "Empirical analysis of dependence between stations in Chinese railway network," Physica A, vol. 388, no. 14, pp. 2949-2955, 2009.

[7] J. Sienkiewicz and J. A. Holyst, "Statistical analysis of 22 public transport networks in Poland," Physical Review E, vol. 72, no. 4, Article ID 046127, 11 pages, 2005.

[8] C. Von Ferber, T. Holovatch, Y. Holovatch, and V. Palchykov, "Public transport networks: empirical analysis and modeling," The European Physical Journal B, vol. 68, no. 2, pp. 261-275, 2009.

[9] R. Albert, H. Jeong, and A.-L. Barabási, "Error and attack tolerance of complex networks," Nature, vol. 406, no. 6794, pp. 378-382, 2000.

[10] P. Holme, B. J. Kim, C. N. Yoon, and S. K. Han, "Attack vulnerability of complex networks," Physical Review E, vol. 65, no. 5, Article ID 056109, 14 pages, 2002.

[11] H. Jeong, S. P. Mason, A.-L. Barabási, and Z. N. Oltvai, "Lethality and centrality in protein networks," Nature, vol. 411, no. 6833, pp. 41-42, 2001.

[12] J. A. Dunne, R. J. Williams, and N. D. Martinez, "Network structure and biodiversity loss in food webs: robustness increases with connectance," Ecology Letters, vol. 5, no. 4, pp. 558-567, 2002.

[13] M. E. J. Newman, S. Forrest, and J. Balthrop, "Email networks and the spread of computer viruses," Physical Review E, vol. 66, no. 3, Article ID 035101, 4 pages, 2002.

[14] R. Cohen, K. Erez, D. Ben-Avraham, and S. Havlin, "Resilience of the Internet to random breakdowns," Physical Review Letters, vol. 85, no. 21, pp. 4626-4628, 2000.

[15] R. Cohen, K. Erez, D. Ben-Avraham, and S. Havlin, "Breakdown of the internet under intentional attack," Physical Review Letters, vol. 86, no. 16, pp. 3682-3685, 2001.

[16] B. K. Mishra and A. K. Singh, "Two quarantine models on the attack of malicious objects in computer network," Mathematical Problems in Engineering, vol. 2012, Article ID 407064, 13 pages, 2012.

[17] M. Li and W. Zhao, "Visiting power laws in cyber-physical networking systems," Mathematical Problems in Engineering, vol. 2012, Article ID 302786, 13 pages, 2012.

[18] M. Hua, P. Cheng, J. Fei, J. Zhang, and J. Chen, "Networkbased robust $H_{\infty}$ filtering for the uncertain systems with sensor failures and noise disturbance," Mathematical Problems in Engineering, vol. 2012, Article ID 945271, 19 pages, 2012.

[19] M. Li and W. Zhao, "On 1/f noise," Mathematical Problems in Engineering, vol. 2012, Article ID 673648, 23 pages, 2012.

[20] V. Latora and M. Marchiori, "Efficient behavior of small-world networks," Physical Review Letters, vol. 87, no. 19, Article ID 198701, 4 pages, 2001.

[21] A. E. Motter and Y. Lai, "Cascade-based attacks on complex networks," Physical Review E, vol. 66, no. 6, Article ID 065102, 4 pages, 2002.

[22] J. Wang and L. Rong, "Cascade-based attack vulnerability on the US power grid," Safety Science, vol. 47, no. 10, pp. 1332-1336, 2009. 
[23] Y. Xia, J. Fan, and D. Hill, "Cascading failure in Watts-Strogatz small-world networks," Physica A, vol. 389, no. 6, pp. 1281-1285, 2010.

[24] M. Babaei, H. Ghassemieh, and M. Jalili, "Cascading failure tolerance of modular small-world networks," IEEE Transactions on Circuits and Systems II, vol. 58, no. 8, pp. 527-531, 2011.

[25] J. Wang and L. Rong, "Edge-based-attack induced cascading failures on scale-free networks," Physica A, vol. 388, no. 8, pp. 1731-1737, 2009.

[26] B. Mirzasoleiman, M. Babaei, M. Jalili, and M. Safari, "Cascaded failures in weighted networks," Physical Review E, vol. 84, no. 4, Article ID 046114, 8 pages, 2011.

[27] S. V. Buldyrev, R. Parshani, G. Paul, H. E. Stanley, and S. Havlin, "Catastrophic cascade of failures in interdependent networks," Nature, vol. 464, no. 7291, pp. 1025-1028, 2010.

[28] C. von Ferber, T. Holovatch, and Y. Holovatch, "Attack vulnerability of public transport networks," in Traffic and Granular Flow '07, pp. 721-731, Springer, Berlin, Germany, 2007.

[29] B. Berche, C. Von Ferber, T. Holovatch, and Y. Holovatch, "Resilience of public transport networks against attacks," The European Physical Journal B, vol. 71, no. 1, pp. 125-137, 2009.

[30] R. Dorbritz and U. Weidmann, "Stability of public transportation systems in case of random failures and intended attacksa case study from Switzerland," in Proceedings of the 4th IET International Conference on Systems Safety Incorporating the SaRS Annual Conference, pp. 1-6, 2009.

[31] J. Zhang, X. Xu, L. Hong, S. Wang, and Q. Fei, "Networked analysis of the Shanghai subway network, in China," Physica A, vol. 390, no. 23-24, pp. 4562-4570, 2011.

[32] Y. Dang, F. Ding, and F. Gao, "Empirical analysis on flight flow network survivability of China," Journal of Transportation Systems Engineering and Information Technology, vol. 12, no. 6, pp. 177-185, 2012.

[33] J. J. Wu, Z. Y. Gao, and H. J. Sun, "Model for dynamic traffic congestion in scale-free networks," Europhysics Letters, vol. 76, no. 5, pp. 787-793, 2006.

[34] J. J. Wu, H. J. Sun, and Z. Y. Gao, "Cascading failures on weighted urban traffic equilibrium networks," Physica A, vol. 386, no. 1, pp. 407-413, 2007.

[35] J. Zheng, Z. Gao, and X. Zhao, "Modeling cascading failures in congested complex networks," Physica A, vol. 385, no. 2, pp. 700-706, 2007.

[36] X. Yang, B. Wang, S. Chen, and W. Wang, "Epidemic dynamics behavior in some bus transport networks," Physica A, vol. 391, no. 3, pp. 917-924, 2012.

[37] K. Lee, W. Jung, J. S. Park, and M. Y. Choi, "Statistical analysis of the Metropolitan Seoul Subway System: network structure and passenger flows," Physica A, vol. 387, no. 24, pp. 6231-6234, 2008.

[38] H. Soh, S. Lim, T. Zhang et al., "Weighted complex network analysis of travel routes on the Singapore public transportation system," Physica A, vol. 389, no. 24, pp. 5852-5863, 2010.

[39] A. L. Huang, W. Guan, B. H. Mao, and G. Z. Zang, "Statistical analysis of weighted complex network in Beijing Public Transit Routes System based on passenger flow," Journal of Transportation Systems Engineering and Information, vol. 13, no. 6, pp. 198204, 2013.

[40] Y. Sui, F. Shao, R. Sun, and S. Li, "Space evolution model and empirical analysis of an urban public transport network," Physica A, vol. 391, no. 14, pp. 3708-3717, 2012.

[41] A. Barrat, M. Barthélemy, R. Pastor-Satorras, and A. Vespignani, "The architecture of complex weighted networks," Proceedings of the National Academy of Sciences of the United States of America, vol. 101, no. 11, pp. 3747-3752, 2004.

[42] K. Kaneko, "Overview of coupled map lattices," Chaos, vol. 2, no. 3, pp. 279-282, 1992.

[43] X. F. Wang and J. Xu, "Cascading failures in coupled map lattices," Physical Review E, vol. 70, no. 5, Article ID 056113, 2004.

[44] J. Xu and X. F. Wang, "Cascading failures in scale-free coupled map lattices," Physica A, vol. 349, no. 3-4, pp. 685-692, 2005.

[45] D. Cui, Z. Gao, and X. Zhao, "Cascades in small-world modular networks with CML'S method," Modern Physics Letters B, vol. 21, no. 30, pp. 2055-2062, 2007.

[46] D. Cui, Z. Gao, and X. Zhao, "Cascades with coupled map lattices in preferential attachment community networks," Chinese Physics B, vol. 17, no. 5, pp. 1703-1708, 2008.

[47] Z. J. Bao, Y. J. Cao, L. J. Ding, G. Z. Wang, and Z. X. Han, "Synergetic behavior in the cascading failure propagation of scale-free coupled map lattices," Physica A, vol. 387, no. 23, pp. 5922-5929, 2008.

[48] C. Di, G. Zi-You, and Z. Jian-Feng, "Tolerance of edge cascades with coupled map lattices methods," Chinese Physics B, vol. 18, no. 3, pp. 992-996, 2009.

[49] X. G. Chen, J. Zhou, and Z. T. Zhu, "Cascading failures study of urban traffic system based on CML," Mathematics in Practices and Theory, vol. 39, no. 7, pp. 79-84, 2009.

[50] X. B. Lu and B. Z. Qin, "Cascading failures in weighted heterogeneous coupled map lattices," in Proceedings of the 1st International Conference on Sustainable Power Generation and Supply (SUPERGEN '09), pp. 1-4, April 2009. 


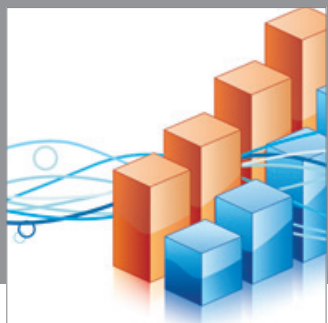

Advances in

Operations Research

mansans

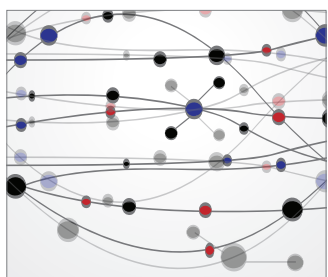

The Scientific World Journal
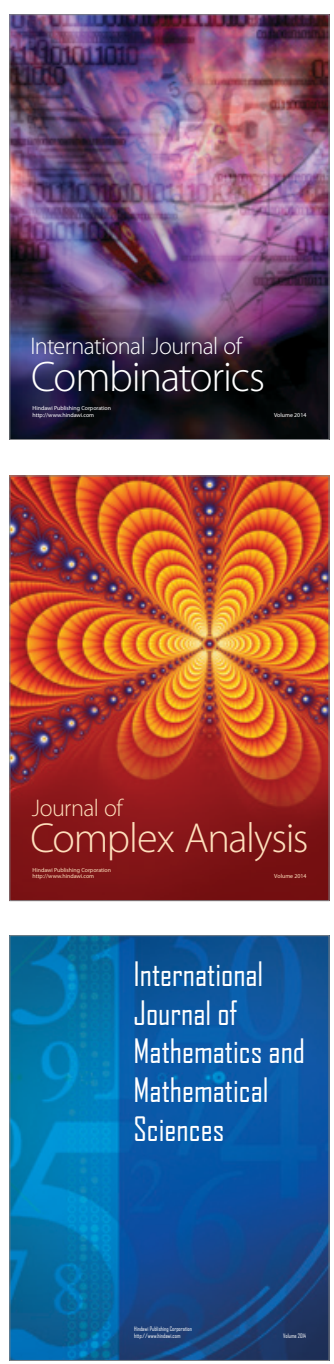
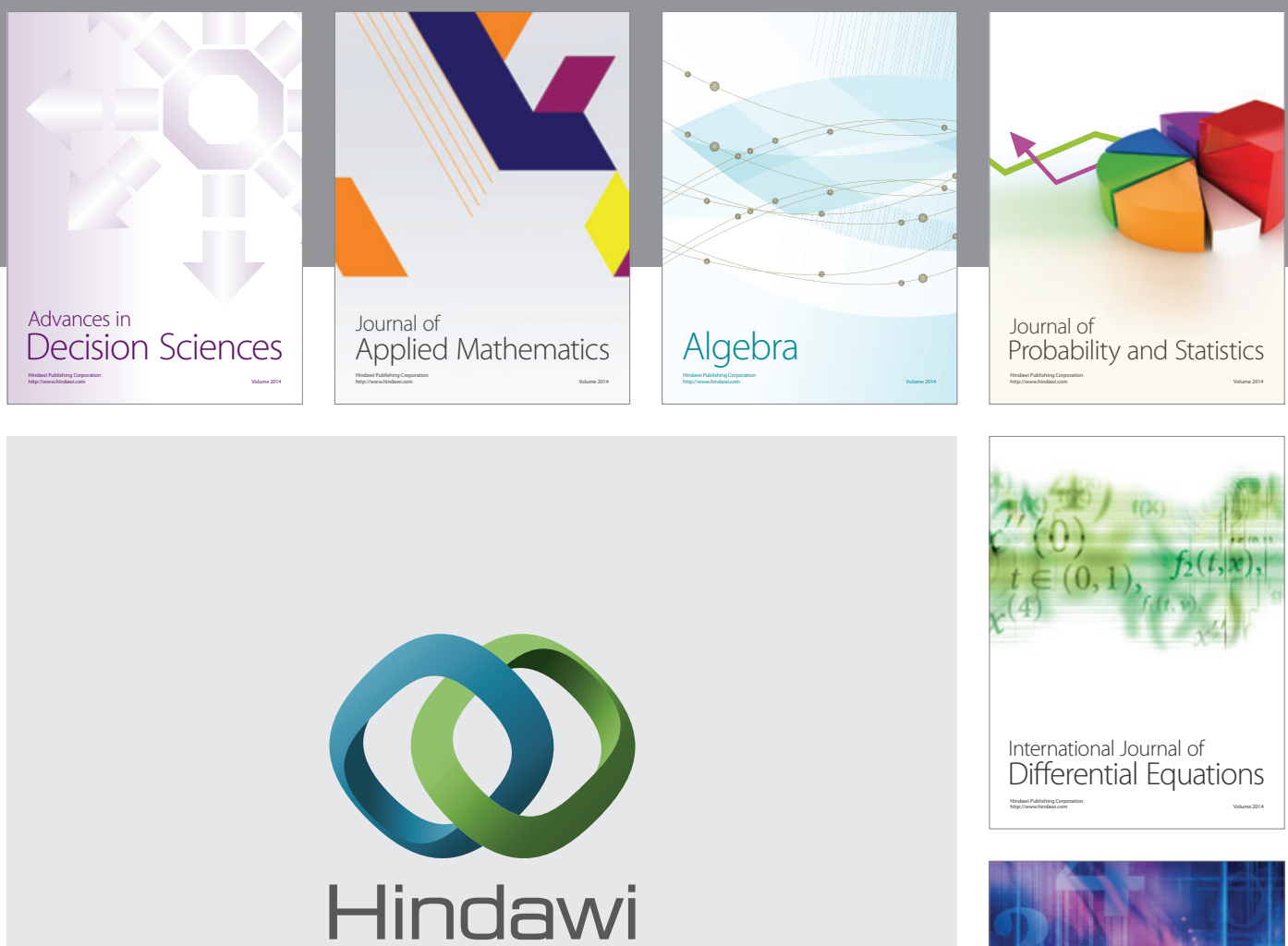

Submit your manuscripts at http://www.hindawi.com
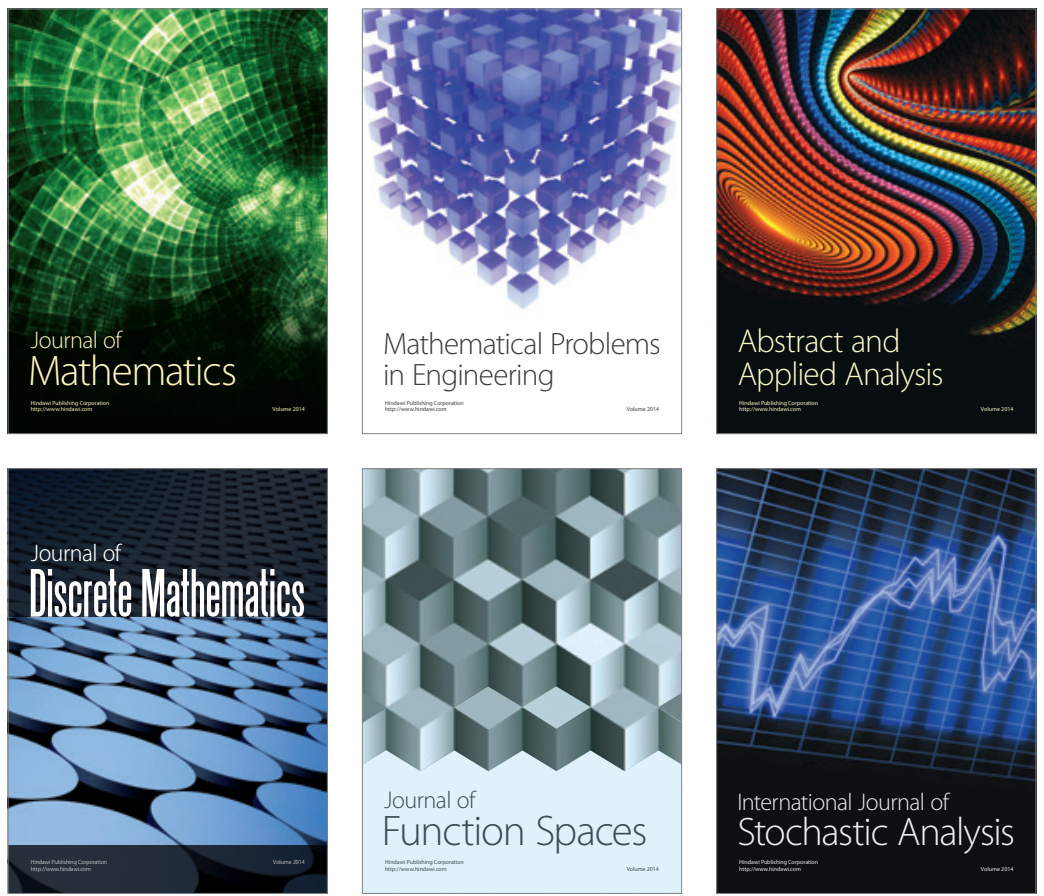

Journal of

Function Spaces

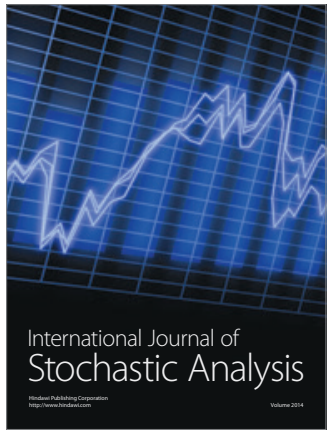

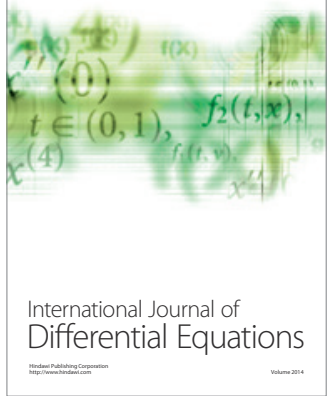
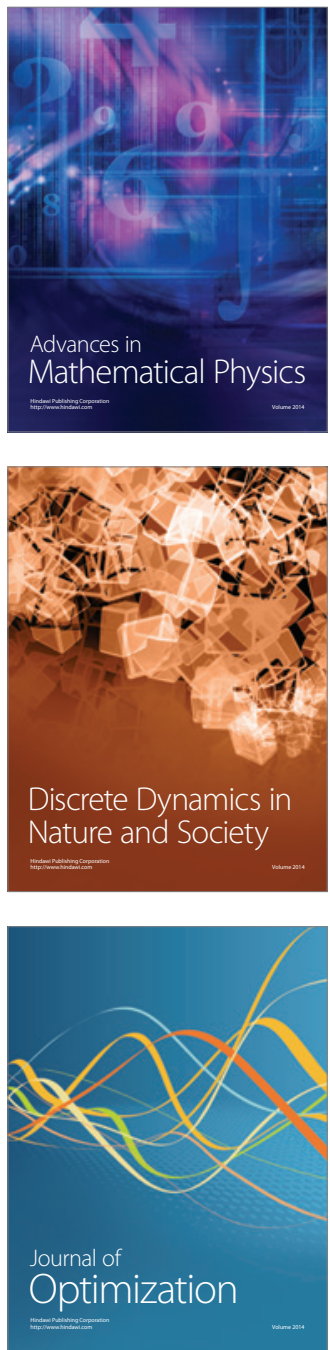